\title{
ACTA ROMANORUM PONTIFICUM IN ARCHIVO \\ DIPUTATIONIS VALENTIAE ASSERVATA
}

(Ab anno MCDX usque ad annum MDCCCXXVI edicta)

POR

M. ${ }^{a}$ Luz Mandingorra Llavata

Universitat de València

Francisco José Sanchis Moreno

Arxiu de la Diputació de València

\section{RESUMEN}

Regesto y estudio de los manuscritos pontificios en pergamino que se conserva en el Archivo de la Diputación de Valencia, que proceden del antiguo archivo del Hospital General de Valencia y del fondo de la duquesa de Almodóvar.

\begin{abstract}
Acta romanorum pontificium in archivo diputationis valentiae asservata.

Digest and study of the pontificial parchment Manuscripts who are kept now on the «Archivo de la Diputación de Valencia» and proceed from the "Antiguo Archivo del Hospital General de Valencia» and the funds from the duchesse of Almodóvar.
\end{abstract}

El interés por la documentación pontificia no es nuevo. Los proyectos de edición de documentos del Papado se remontan a mediados del siglo XIX, cuan-

La Personalidad internacional de los Papas Hispania Sacra 48 (1996) 
do Philippe Jaffé publicó los Regesta pontificum Romanorum', cuya segunda edición corrió a cargo de Wilhelm Wattenbach, Stefan Löwenfeld, Friedrich Kaltenbrunner y Paul Ewald ${ }^{2}$. Por su parte, entre 1874 y 1875, August Potthast había continuado la obra de Jaffè con los Regesta pontificum Romanorum inde ab anno post Christum natum MCXCVIII ad annum MCCCIV. Se iniciaba así una serie de publicaciones que proseguiría durante toda la primera mitad del siglo XX, sin embargo, sólo en 1952 se puso de manifiesto la conveniencia de elaborar un censo general de los documentos pontificios, particularmente, de los medievales ${ }^{3}$. En 1978 la Commission Internationale de Diplomatique emprendió un proyecto titulado Index actorum romanorum pontifictm $^{4}$, que se proponía dar noticia de todos los documentos pontificios editados o inéditos, conservados en bibliotecas y archivos públicos y privados, ya se tratara de originales o de copias. Con este objetivo se formuló un programa de publicación que incluía una serie de normas relativas a la presentación del catálogo, y que afectaban a la regesta, la descripción de las piezas y la confección de los índices 5 .

Aunque los documentos que aquí presentamos superan con mucho los límites cronológicos establecidos en el proyecto de la Comisión, hemos considerado necesario, por una cuestión de coherencia, aplicar al conjunto de la documentación la normativa propuesta, ya que no era posible tratar los documentos modernos de un modo diferente a los medievales, sin crear una distorsión, puesto que todos ellos se integran en el mismo fondo documental y constituyen parte de la memoria de la misma institución.

1 AFFÉ, PH.: Regesta pontificum Romanomm ab condita Ecclesia ad ammu post Christam natum 1/98, Bertín 1851.

2 Berlín 1885-1888.

" Bartoloni, F.: "Per un censimento dei documenti pontificii da innocenzo III a Martino V escluso". En La publicazione delle fonti del medioevo earopeo negli uhini 70 aani, 1883-1953. Roma, 1953; debe verse además BATELL, G.: "Documento pontificio". En Boletín de la Sociedad Castellonetuse de Culura, LVIII (1982), pp. $571-627$

- COMMISSION INTERNATIONALE DE DIPLOMATIQUE: Index actormm romahorum pomificum, Ab hinocentio $/ /$ usque ad Martinum $V$ electum qua praeter registra romanae curiae adservanfu. 1978

5 Estas normas fueron utilizadas por Barbiche, BARBICHE, B.: Les Actes Ponificatix originaus des Archives Nationales de Paris. 3 vols. Paris, 1975-1982, y, ya en España, por SERRA EsTELLÉS, X.: Acta Romanorum Pontificum in Archivo Regni Valentini asservata (Ab anno Christi MLXXIV usque ad amum MDCIV edicta). Valencia, 1981; Pozo CHACón, J. A.: Acta Romanortm Pontificum (132/-14/4) quae in Archivo Cahedralis Valentinae asservantur. Tesis de licenciatura inédita, Valencia, 1980, y por GonZÁleZ BALASCH, M." T.-FERNÁNDEZ DE VIANA Y Vieites, J.L.: "Lai documentación pontificia en el 'tumbo B' de la catedal de Suntingo". En Miscel. iñia d'estudis dedicats a la memoria del professor Josep Trenchs i Ödena, Castelló, 1995 (Estudis Castellonencs, VI. l), pp. 627-646.

La Personalidad Internacional de los Papas

Hispania Sacra 48 (1996) 
El número de documentos pontificios que en la actualidad conserva el Archivo de la Diputación de Valencia ${ }^{6}$ asciende a un total de sesenta y nueve, y en su mayor parte, han permanecido inéditos hasta hoy. De hecho, las numerosas referencias y ediciones de los llamados privilegios fundacionales del, en primer lugar, Hospital de Inocentes y, más tarde, del Hospital General de Valencia, se han realizado, en la mayoría de los casos, sobre diferentes copias conservadas en otros archivos de la ciudad, y rara vez a partir de los originales.

El fondo de pergaminos conservado en el Archivo de la Diputación de Valencia tiene un doble origen, aunque motivos históricos, a los que aludiremos más tarde, han provocado su confluencia. Se trata de los dos únicos fondos allí custodiados que contienen documentación pontificia: por una parte, el archivo histórico del actual Hospital General de Valencia, heredero del que se fundara en 1410, bajo la denominación de Hospital de los Santos Inocentes ${ }^{7}$, y por otra, el fondo de la Duquesa de Almodóvár, cuya documentación más antigua también se remonta a fines de la Edad Media.

La circunstancia histórica que posteriormente unió físicamente a estos dos archivos, el primero público - aunque en su origen fue privado- y el segundo, netamente familiar, fue la muerte de doña Josefa Dominga Català de Valleriola, Duquesa de Almodóvar, Marquesa de Ontiveros, Nules y Quirra, Condesa de Canalejas, de L'Alcúdja y Gestalgar, Baronesa de Sot de Xera, Xalon, Estivella y Tous. Su fallecimiento se produjo en 1813, pero ya en su testamento, formalizado en 1804, declaró como heredera a su alma, para cuyo sufragio ordenó que las rentas obtenidas por la administración de sus bienes o la venta de los mismos, se distribuyeran entre sus vasallos huérfanos próximos a contraer matrimonio. Este legado llevaría, finalmente, a que la administración de esta testamentaría recayera en la Junta Provincial de Beneficencia, que, a su vez, ordenó que dichos fondos se incorporasen a la Diputación por un decreto de 30 de septiembre de $1868^{8}$. Así pues, este fondo pasaba, al menos en teoría, a estar bajo la custodia de la Diputación de Valencia, al igual que el del Hospital General, que adquirió carácter provincial en 1850 . Su ingreso físico en el A.D.P.V no se produjo hasta bien entrado el siglo XX.

El conocimiento en profundidad de estos fondos se inicia con la noticia de los relativos al Hospital de General de Valencia que se publicó en 1971, donde el número de pergaminos que se cita dista mucho de los más de 4.000 actual-

\footnotetext{
6 En adelante se citará mediante las siglas A.D.P.V.

7 En cuanto a la evolución y cuestiones históricas relativas al Hospital General remitimos a la bibliografía citada al final de este artículo

в Vid. Guía del Archivo de la Excma. Diputación Provincial de Valencia. Valencia, 1990, pp. 176-187.
} 
mente inventariados en dicho fondo, y donde no se nombra ningún documento pontificio ${ }^{9}$. Por lo tanto, las noticias referentes a los mismos, al igual que los relativos al archivo nobiliario, proceden de la bibliografía clásica relativa al Hospital General y por la Guía del Archivo de la Diputación que se publicó en 1990.

Además de los pergaminos, el fondo del Hospital en el Archivo de la Diputación conserva en la actualidad dos cartularios y un bulario en los que se transcriben diversos documentos pontificios. El más antiguo ${ }^{10}$ recoge las constituciones del Hospital de Inocentes, así como los privilegios reales y bulas que determinaron su erección. Data de comienzos del siglo XV, cuando se inicia la actividad de dicho hospital, aunque su proceso de escrituración se prolonga hasta la segunda mitad del siglo XVII, ya que el libro era constantemente reutilizado por los distintos administradores y gestores del centro hospitalario. A esta fecha se remonta el segundo cartulario, el Llibre de constitucions, ordinacions e indults apóstòlics concedits en favor de l'Espital General de València ${ }^{11}$. Es copia de un libro anterior, indudablemente del $0 / 1$, tal como se indica en una apostilla junto al título: Les presents constitucions son estades tretes del libre antich que està en lo archilu, desde el fol. 16 hasta el fol. 47 y desde el fol. 171 asta el fol. 186 y desde el fol. 192 asta el fol. 206. Finalmente encontramos el Copiador de Bulas Apostólicas. Año $1781^{12}$. La primera bula transcrita es de 1775 , si bien la bula más antigua registrada, data del 22 de marzo de 1774; por su parte, la más reciente lleva fecha de 26 agosto de 1826.

- Casal Novoa, F.: "Noticia de los fondos documentales existentes en el Archivo del Hospital General de la ciudad de Valencia". En / Congreso de Historia del País Valenciano, I, Valencia, 1973, pp. 411-427.

10 A.D.P.V., Hospital General, libros, 0/1. Se trata de un volumen de gran formato (400x303 $\mathrm{mm}$ ), compuesto por $204 \mathrm{ff}$. en papel, con doble foliación, una de la ćpoca, en números romanos, y otra arábiga actıal. El primer folio desapareció, razón por la que no conocemos el título original que se dio al libro. Recientemente ha sido objeto de una restauración, por lo que presenta una encuadernación actual de cuero repujado. Los ff. 7 v. a 13 v., 71 y 109 a 113 aparecen en blanco. Cabe sē̄alar, como curiosidad, que aparecen diferentes anotaciones y asientos de mano de Jaume Roig, que fue administrador del Hospital de Inocentes.

"I A.D.P.V., Hospital general, libros, 0/2. Ese es el título que aparece en el f. $8 \mathrm{r}$. Se trata de un volumen algo mós pequeño que el anterior $(335 \times 238 \mathrm{~mm})$. Se halla constituido por $238 \mathrm{ff}$, de papel, con guardas de pergamino. Presenta dos foliaciones, la primera, de la época, en números arábigos, corregida. La segunda, una foliación actual, en lápiz, Los ff. 112 v. a 161 v. y 171 r. a 238 v. se hallan en blanco. Por su parte, los folios 168 y 170 son dos cartas reales fechadas en 1688 y 1697 respectivamente. La encuadernación es de cuero repujado.

12 A.D.P.V., Hospital General, libros, IV-4, I/2. El bulario se halla constituido por $37 \mathrm{ff}$. de papel, con unas dimensiones de $341 \times 241 \mathrm{~mm}$. La foliación es arábiga, de la épocn. Los ff. 32 a 37 se hallan en blanco. La encuadernación es de pergamino con cierres de cáñamo hoy perdidos.

La Personatidad Internacional de los Papas

Hispania Sacra 48 (1996) 
En la descripción subsiguiente se han obviado todas aquellas notas que en el transcurso de los siglos se insertaron en el dorso de los documentos, por tratarse de anotaciones de carácter archivístico. Normalmente, se trata de regestas y signaturas antiguas, sobre las cuales creemos aconsejable realizar algunas precisiones:

- Las bulas del siglo XV y las de comienzos del XVI suelen llevar al dorso un extracto, que, a través del análisis paleográfico, puede datarse como coetáneo al documento, y otro posterior que podríamos fechar a fines del XVII o comienzos del XVIII. Este se haría necesario a causa de la dificultad de lectura que presentaban las regestas primigenias, muchas de las cuales resultan hoy casi ilegibles por lo desvaído de las tintas empleadas.

- La mayoría de las bulas del Hospitál General, hasta el pontificado de Sixto $\mathrm{V}$, presentan una signatura antigua consistente en la fórmula $B$. $n$, que hay que entender como Bulla número... ${ }^{13}$ Lo que nos hizo pensar que, probablemente, hubo inicialmente en el archivo del hospital, un estante o cajón que diferenció a este tipo documental de otros que pudieran hallarse entre los pergaminos ${ }^{14}$. Afirmación que quedó plenamente confirmada al citarse en un inventario del archivo ${ }^{15}$, realizado en 1668: "una caixa de privilegis y bulles apostòliques". Lo cual se corrobora además al inspeccionar los privilegios reales, que en su dorso presentan igualmente una signatura antigua $P . n$. (=Privilegi número...), escrita por la misma mano que la que aparece en las bulas.

Con esta misma signatura, B. n., aparece junto a las bulas, un documento pontificio no papal ${ }^{16} \mathrm{en}$ el que los cardenales que integran la Confraternitas et

13 Existen algunas excepciones, como la bula de Benedicto X11!, datada el 27 de agosto de 1411 , sobre la que no fue incluida esta nota sin que exista un motivo aparente para ello.

14 Actuaimente, dentro de los pergaminos, no hay distinción por tipos documentales sino que se ha aplicado un número currens a la totalidad de los documentos que aparecen sobre esta materia escriptoria.

15 A.D.P.V., Hospital General, IV $4.5 / 2$, f. 40.

i6 A.D.P.V., Hospital General, perg. 3343 (1590 febr. 4. Roma). Aunque en el inventario del archivo (IV-4.5/2, f. 4l) se la describe como: "Una bulla de Sixto quinto en que concedeix los mateixos privilegis que estan concedits al Hospital de Santiago en Roma, sub nt ${ }^{\circ} 36^{\prime \prime}$, сп concreto se trata de una indulgencia colectiva, ya que presenta todas las características que Thomas Frenz fija para esta tipología por lo que se refiere a: intitulación, dirección, salutación, datación y disposición de las notas de cancillerín. También aparecen otras características que son propias de este tipo documental, como es la decoración pintada en los márgenes superior, izquierdo y derecho, y la utilización como tipos gráficos de la capital humanística y ja humanística cursiva. Cfr. FRENZ, THOMAS: I documeni pomi- 
Societas Archihospitalis Sancti lacobi Incurabilium de Roma conceden al Hospital General todas las indulgencias, privilegios e indultos otorgados a su institución por diversos pontífices.

\section{REGESTA DOCUMENTORUM PONTIFICUM ${ }^{17}$}

BENEDICTUS (XIII) "dilectis filiis Vallisdign., Valentin. dioc., Sancti Bernardi [extra]muros Valentin., monasteriorum abbatibus ac officiali Segobricen.": concedit licentiam administratoribus hospitalis pauperorum, quod Sanctorum Innocentium nuncupatur in civitate Valentin., ut capellam et cimiterium in dicto hospitale aedificent, et hoc modo pauperibus et infirmis omnia ecclesiastica sacramenta dare ac etiam sua corpora sepelliri posssint. Dat. Barchinonae, IV kal. mart., pont. a. XVI. "Sincerae devotionis affectus".

Valencia, A.D.P., Hospital General, perg. 11. Jhs. in publ. instr. 16 meii 1410. Describitur cum filo can.

Valencia, A.D.P., Hospital General, 0/1, f. XX r. - v. et iterum in f. XXI r. - v. Cop. in chartulario s, $X V$.

Valencia, A.D.P., Hospital General, 0/2, if. $9 \mathrm{r},-10 \mathrm{r}$, et iterum in f. $/ / \mathrm{v} .12 \mathrm{r}$. Cop. in chartulario s. XVII.

Valencia, A.D.P., Hospital General, JV-4.2, Icg. 9. Bulla inpressa in chalcographia Josephi Thomae Lucas, qui typographus ab 1736 usque 1774 fuit.

Ed.: J. Rodrigo Pertegás, Obra histórica de la ...Cofradía de Ntra. Sra. de los Mocentes, apéndice I, pp. 469-472, documentum editum transcriptum fuit profectus non ab originale, sed a cop. not., 3 oct. 1410, in Archivio Cathedralis Valentiae asservata (sig. 3569 , f. 278 v.). - Cf.: H. Tropé, Locura y sociedad en la Valencia de los siglos XV al XVII, p. 59.

BENEDICTUS (XIII) "ad perpetuam rei memoriam": concedit auctoritatem administratori Hospitalis Sanctorun! Innocentium, ut omnia legata, donationes, oblationes ac elemosynas accipere possit, ac etiam voluit quod ipse administrator hospitalis pro jure illi parrochiali ecclesiae, ad quam pro tempore pertinebit, anno quolibet duos

fici nel medioevo e nelletà moderna. Ed. italiana a cura di S. Padano, Città del Vaticano, 1989, pp. 94-95.

17 Nomina personarum et locorum servando documentorum graphiam transcripta sunt.

La Personalidad Internacional de los Papas

Hispania Sacra 48 (1996) 
florenos auri solvere debeat. Dat. Barchinonae, IV kal. mart., pont. a. XVI. "Ex iniuncto nobis" - "constitutionis, inhibitionis et voluntatis".

Valencia, A.D.P., Hospital General, 0/1, If. XXI y. - XXII r. Cop. in chartulario s. XV. Describitur cun filo ser.

Valencia, A.D.P., Hospital General, 0/2, ff, 12 v. - 13 r. Cop, in chartulario s. XVII.

Valencia, A.D.P., Hospital General, IV-4.2, leg. 9. Bulla impuessa in chalcographia Josephi Thomae Lucas, qui typograp/us ab 1736 usque 1774 fuit.

Ed.: J. Rodrigo Pertegás, Obra histórica de la ... Cofradía de Ntra Sra. de los Inocentes, apéndice II, pp. 473 - 475, documentum editum transcriptum fuit profectus non ab originale, sed a cop. not. , 3 oct. 1410, in Archivio Cathedralis Valentiae asscrvata (sig. 3569, f. 490), - Cf.: H. Tropé, Locura y sociedad en la Valencia de los siglos XV al XVII, p. 59.

BENEDICTUS (XIII) "Universis Christi fidelibus presentes litteras inspecturis": concedit Ferdinando Garsie, Johanni Armenguer, Petro Çaplana, Francisco Barcelo, Jacobo Dominguez, Stephano Valenca, Sancio Calbo, Bernardo Andree, Petro Pedrea et Petro de Bovia licentiam ut capellam aedificent in hospitali pauperorum, quod Sanctorum Innocentium nuncupatur in civitate Valentin., et indulgentias tabellionibus qui testamenta recipiendo hospitale predictum et eius pauperes recomendaverint, necnon omnibus fidelibus qui dictam capellam in constitutis festivitatibus visitaverint. Dat. Barchinonae, IV kal. mart,, pont. a. XVI. "Quoniam, ut ait apostolus".

Valencia, A.D.P., Hospital General, perg. 10. Originale. Bulla dep. Recto. super plicam in ang. sup. ad d.: Gratis de mandato domint nostri papae / Laurentits et prope : n sub plicat ad s. taxa : Gratis de mandato domini nostri papae / A. de Campis sub plica in ang. inf. ad s.: Expedita V1 id. apr. a. XVI B. Fortis sub plica ad d.: G. proton. Lucas de Neapoli A tergo, in ang. sup. ad s.: Expedita VI id. apr. in media pagina.

Ed.: J. Rodrigo Pertegás, Obra histórica de la ...Cofradía de Ntra. Sra. de los hocentes, apéndice IV, pp. 481-482. (Reg. et imago photographica: F. Donzingo, El primer hospital psiquiátrico del mundo, p. 55 et doc. 5. (Imago photographica: E. M. Aparicio, Santa María de los Inocentes y Desamparados, p. 355. ( Cf. H. Tropé, Locura y sociedad en la Valencia de los siglos XV al XVII, p. 59.

BENEDICTUS (XIII) "dilectis filiis Ferdinando Garciae, Joanni Armenguer, Petro Çaplana, Francisco Barcelo, Jacobo Dominguez, Stephano Valença, Sanccio Calbo, Bernardo Andreae, Petro Pedrera et Petro de Bonia, civibus Valentin": concedit condi- 
toribus et primo hospitalario hospitalis pauperum, quod Sanctorum Innocentium nuncupatur in civitate Valentin., et uxoribus suarum in mortis articulo plenam remissionem peccatorum suorum. Dat. Barcinonae, IV kal. mart., pont. a. XVI. "Provenit ex nostrae devotionis" - "concessionis et voluntatis".

Valencia, A.D.P., Hospital General, 0/1, f. XXIV r. - v. Cop. in chartulario s. XV. [Recto, super plicam ad d.: Joannes Belier sub plica ad s. taxa: Gratis de mandato domini nostri papae / A. de Campis sub plica ad d.: G. proton. Lucas de Neapoli I.

Valencia, A.D.P., Hospital General, 0/2, ff. $16 \mathrm{v},-17 \mathrm{v}$. Cop. in chartulario s. XVII.

BENEDICTUS (XIII) "dilecto filio Iohanni Compayn, perpetuo beneficiato in parrochiali ecclesia Sancti Michaelis de Cardona, Urgeliensis dioc.": constituit eum rectorem parrochialis ecclesiae de Maldano, Terraconen. dioc.. Dat. Terraconae, II id. sept., pont. a. XVI, "Vitae ac morum honestas" - "reserva-tionis, inhibitionis, constitutionis et voluntatis".

Valencia, A.D.P., Hospital General, perg. 3836. Originale, cum aliquis foraminibus. Bulla non fuit appensa, cum foramina desint. Cancellatus. Recto, in margine sup.: non comput. ( non comput. Recto, ad s.: unum / per quas tibi de (...) beneficio provideri mandamus ut prefertue ad d.: G. super plicam ad d.: Expedita gratis / Laurentius sub plica, ad s. taxa : XII (in columna) A tergo: N. Conilli n.

BENEDICTUS (XIII) "dilecto filio Petro Clavelli": conferit et providit ei vicariam ecclesiae parrochialis B. Mariae Magdalenae Cesaraugustae, cum cura animarum. Dat. apud S. Matheum, Dertusen. dioc., VI id. aug., pont. a. XVII. "Litterarum scientia" "collationis, provisionis, constitutionis et voluntatis".

Valencia, A.D.P., Hospital General, perg. 2403. Originale. Bulla dep. Recto in margine sup. ad d.: G. super plicam ad d.: Expedita gratis /M, de Ceresuela sub plica ad s. taxa : XIII in columna) / Expedita VI kal. sept. a. XVII A. de Campis A tergo in media pagina; in margine inf.: graciosa domini Petri Clavelli in margine sup.: $\mathrm{D} / \mathrm{C}$.

BENEDICTUS (XIII) "universis Christi fidelibus presentes litteras inspecturis": relaxat centum dies eis omnibus qui pro remedio animarum defunctorum Christi fidelium iudicialiter suspensorum in civitate Valentin., missas celebraverint, aut preces vel oraciones, ut missae factae erint, fecerint, aut corpora vel ossa associaverint. Dat. apud S. Matheum, Dertusen. dioc., VI kal. sept., pont. a. XVII. "Quoniam, ut ait apostolus".

La Personalidad Internacional de los Papas

Hispania Sacra 48 (1996) 
Valencia, A.D.P., Hospital General, perg, 3349. Originale. Bulla dep. Recto, super plican ad d.: Expedita gratis / M. de Ceresuela sub plica ad s. iaxa ; XVI (in columna) / Expedita X kal. oct., a. XVII A. de Campis ad d.: F. Aurien. A. de Belencinis, n. A tergo in media pagina:

Valencia, A.D.P., Hospital General, 0/1, ff. XXIII v. -XXIV r. Cop. in chartulario s. XV.

Valencia, A.D.P., Hospital General, 0/2, f. 16 r. - v. Cop. in chartulario s. XVII. Describitur cum filo ser.

BENEDICTUS (XIII) "AD PERPETUAM REI MEMORIAM": concedit licentiam administratoribus Hospitalis Sanctorum Innocentium in civitate Valentin. capellam usque ad centum palmos in longitudine et quinquaginta palmos in latitudine, cum uno altare sub invocatione $\mathrm{S}$. Crucis Montis Calvari aedificare ac etiam in cimiterio dicti hospitalis corpora infirmorum, qui decedant in eodem ac etiam cadavera iudicialiter suspensorum, sepelliri possint. Dat. apud Sanctum Matheum, Dertus. dioc., VI kal., pont. a. XVII. "Pia fidelium vota" - "concessionis, statuti et ordinationis".

Valencia, A.D.P., Hospital General, perg. 2263. Originale. Membrana mutila cum magnis foraminibus et cum rasis et emendatis. Bulla dep. Recto, super plicam ad d.: M. de Ceresuela et prope: V. sub plica ad s. taxa: LX / F. Aurien. sub plica in ang. inf. ad s.: Expedita XVIII kal.. oct. a. XVII A. de Campis /. A . sub plica ad s.: [G.] proton. A. Belencinis A tergo, in media pagina: Rta.

Valencia, A.D.P., Hospital General, perg. 7. Cop. in privilegio Ferdinandi II, regis Aragonum, 5 dec. 1493.

Valencia, A.D.P., Hospital General, 0/1, ff. XX v. - XXIII v. Cop, in chartulario s. XV. Describitur cum filo ser.

Valencia, A.D.P., Hospital General, 0/2, ff. 14 v. - 16 r. Cop. in chartulario s. XVII Describitur cum filo ser.

Ed.: J. Rodrigo Pertegás, Obra histórica de la... Cofradia de Nrra. Sra de los Inocentes, apéndice III, pp. 476-480, documentum editum transcriptum fuit profectus non ab originale, sed a registro cancellariae Ferdinandi II, regis Aragonum, in Archivio Regni Valentiae asservatum (Real Cancillería, 309, f. 203 v.). ( Cf.: E. M. Aparicio, Santa María de los Inocentes y Desamparados, p. 149 (sub data 1417 oct. 24); H. Tropé, Locura y sociedad en la Valencia de los siglos XV al XVII, p. 58 (sub data 1411 oct. 24).

Benedictus (XIII) "venerabili fratri epo. et dilectis filiis abbati monasterii Sancti Bernardi extra muros Valentin. ac officiali Dertusen.": mandat eis collationem et provisionem perpetui benefici ad altare S. Bernardi, situm in ecclesia monasterii de La Çaydia, prope muros Valentin. , cisterc. ordinis, quod vacat per obitum Ludovici Sala, 
in favorem Bernardi Ras, beneficiati in ecclesia Valentin. Dat. in Peniscole, Dertusen. dioc., X kal. aug., pont. a. XVIII. "Vitae ac morum honestas".

Valencia, A.D.P., Hospital General, perg. 3835. Originale. Bulla dep. Recto, in margine sup. ad d.: G. super plicam ad d.: Rta. gratis / G. Coma sub plica ad s. taxa: XVI (in columna) / Expedita VI kal. dec. a. XVIII A. de Campis A tergo, in ang. sup. ad s.: Jo. Belier in media pagina:

Benedictus (XIII) "Venerabili epo. Valentin.": committit et mandat ei dispensationem matrimonio inter Bernardum Catalam et Johannetam de Centillis, quae in quarto consanguinitatis gradu sunt. Dat. Paniscole, Dertusen. dioc., II non. iul., pont. a. XIX. "Oblatae nobis".

Valencia, A.D.P., Duquesa de Almod6var, perg. 434. Ins. in publ. instr. 21 iul, 1413. Describitur cum filo can.

EUGENIUS IV "ad perpetuam rei memoriam": indulget, ut confessor personarum quas in Hospitali B. Mariae de Gracia civitatis Cesaraugustae decedere contigerit, et confesse fuerint, plenam remissionem eis concedere valeat. Dat. Romae, apud S. Petrum, a. inc. dom. MCDXXXI, XVIII kal. febr., pont. a. I. "Dum ad illam" "concessionis et voluntatis".

Valencia, A.D.P., Hospital General, perg. 2204. Cop. not. s.d. Describitur cum filo ser. Transcribitur sine loco intra membranam: P. de Magio, Meynardus, C. Meius, Fe. CXXXX G. de Imola, Registrata B. Valven.

Eugenius (IV) "ad perpetuam rei memoriam": validat et habilitat, per concordiam cum rege Alphonso Aragonum, facultates, dispensationes... concessas in dictis regnis per Consilio Basilien. et Amedeo, olim duce Sabaudiae, et mandat omnes et singulae causae, quae ideo existent, inter quascumque personas et subditos prefati regis, ordinariis vel delegatis iudicis pertineant. Dat. Romae, apud S. Petrum, a. inc. dom. MCDXLIV, kal. nov., pont. a. XIV. "Illius qui a Patre" - "absolutionis, habilitationis, restitutionis, reintegrationis, repositionis, communicationis, exceptionis, confirmationis, concessionis, anullationis, constitutionis, collationis, reservationis, mandati, revocationis, cassationis, deputationis et voluntatis".

La Personalidad Internacionas de los Papas

Hispania Sacra 48 (1996) 
Valencia, A.D.P., Duquesa de Almod6var, perg. 1128. Ins. in publ. instr, 16 nov, 1448. Membrana parum mutila cum parvis foraminibus. Describitur cum filo can.

Eugenius (IV) "dilecto filio Francisco Cruilles can. Valentin.": mandat ei, auctoritatern apostolicam super beneficium capellae B. Mariae Hospitalis Sanctorum Innocentium civitatis Valentin. restituere et reponere. Dat. Romae, apud S. Petrum, a. inc. dom. MCDXLVI, [... kal.] nov., pont. a. XVI. "Sedis apostolicae providentia".

Valencia, A.D.P., Hospital General, perg. 3335. Originale, cum aliquis foraminibus et maculis. Butla dep. Recto, super plicam ad s.: Poggius ad d.: pro P. Sessenal /L. de Castiliono sub plica ad s.: oct. et prope taxa: XX (in columna) / Ugolinus / R. A tergo in media pagina: in Camera Apostolica P.

Ed.: F. Domingo, El primer hospital psiqutatrico del mundo, pp. $55-56$ et doc. 6 ; ed. partim E. M. Aparicio, Santa María de los Inocentes y Desamparados, pp. 149 150 (imago photographica p. 374).

NICOLAUS (V) "dilecto filio Johanni Stephani, pres. perpetuo beneficiato in parrochiali ecclesia S. Crucis Valentin.": concedit ei perpetuum beneficium ad altarem S. Barbarae in ecclesia Valentin. Dat. Romae, apud S. Petrum, a. inc. dom. MCDXLVIII, III kal. ian., pont. a. II. "Vitae ac morum honestas" - "concessionis".

Valencia, A.D.P., Duquesa de Almodóvar, perg. 148. Originale, cum aliquis foraminibus. Bulla dep. Recto, super plicam: Registrata (...) L. de Castiliono sub plica ad s. taxa : XXIl (in columna) A tergo: in margine inf.: A. de Cortesiis.

NICOLAUS (V) "dilecto filio abbati monasterii Sancti Bernardi extra muros Valentiae": onerat perpetuum beneficium in ecclesia Hospitalis Sanctorum Innocentium, quod vacat per resignationem Francisci Cabrera, cum Bernardo Rodrigo, presbitero Valentin. Dat. Romae, apud S. Petrum, a. inc. dom. MCDLII, id. ian., pont. a VI. "Dignum arbitramur".

Valencia, A.D.P., Hospital General, perg. 3350. Originale. Bulla dep. Exstat filum can. Recto, in margine ad s.: d. sub plica ad s.: oct. et prope XX (in columna) / A. de Veneriis super plicam ad d.: dupplicata / Jo. de Collis A tergo, intra foramina bullae: pro Nicasio Jac. Bignetti.

Valencia, A.D.P., Hospital General, perg. 1906. Ins. in publ. instr. 17 dec. 1453. Describitur cum filo can.

La Personalidad Internacional de los Papas Hispania Sacra 48 (1996) 
NICOLAUS (V) "AD FUTURAM REI MEMORIAM": restituit et reponet plenarie Agneti de Portugalia, uxori quondam Gundisalvi d'Ixar, et filiis suis, concessiones, donationes et iliarum confirmationes super praeceptorias Montisalbani, vallis de Orthetha, Villae Joyosae, Museros et Enguerae, et statuit et decernet, quod dictas concessiones plenarn firmitatem obtineant. Dat. Romae, apud S. Petrum, a. inc. dom. MCDLIII, VII kal. iun., pont. a. VII. "Solet nimirum Romani Pontifices" - "absolutionis, revocationis, cassationis, annullationis, mandati, restitutionis, repositionis, statuti, constitutionis, impositionis, approbationis, confirmationis, communitionis, supplectionis, derogationis et voluntatis".

Valencia, A.D.P., Duquesa de Almodóvar, perg, 1077. Originale, cum aliquis foraminibus. Bulla non fuit appensa, cum foramina desint. Recto, super plicam: Pro Jo. de Mamen vel Vicentia / L. de Marnia.

Calistus (III) "Dilecto filio officiali Valentin.": decernit omnia et singula privilegia, inmunitates, gratias et indulgentias concessa per pontifices predecessores Hospitali Sanctorum Innocentium vim et vigorem habere, ac firmitate subsistere, ac etiam concedit porrectionem indulgentiarum fidelibus, qui pro conservatione ecclesiae seu capellae necnon sustentatione pauperorum hospitalis adiuvent. Dat. Romae, apud S. Petrum, a. inc. dom. MCDLV, kal. sept., pont. a. I. "Et si ex debito".

Valencia, A.D.P., Hospital General, perg. 12. Originale. Bulla dep. Recto, super plicam ad d.: pro A. Trapezuntio / C. Fidelis sub plica ad s.: aug. et prope taxa: LXX (in columna) / M. Amici sub plica ad d.: M. Joannes A tergo, in medio margine sup.: A. Joannes in media pagina: Rta. [apud me M. Jo]annem.

Cf.: E. M. Aparicio, Santa María de los Inocentes y Desamparados, p. 150.

SIXTUS (IV) "Dilectis filiis Valentin. et Barchinen. ac Dertusen. officialibus" : mandat eis licentiam sollicitent a procuratoribus, qui in nomine monasterii S. Quiteriae virginis, loci de Manso, Aduren. dioc., ordinis S. Benedicti, vota et legata exhigent et levant intra Regnum Valentiae ac Principatum Cathaloniae. Dat. Tibure, a. inc. dom. MCDLXXIII, XIII kal. aug., pont. a. II. "Aut movet nos".

Valencia, A.D.P., Hospital General, perg. 548. Cop. not. 30 aug. 1474. Membrana mutila cum parvis foraminibus. Describitur cum filo can.

La Personalidad Internacional de los Papas

Hispania Sacra 48 (1996) 
Sixtus (IV) "dilectis filiis archidiacono ct Berengario Clavell, canonico ecclesiae Valentin.": constituit eos iudices, comisarii et exequtores in litigio inter Ludovium Dezpuig, magistrum milicie B. Mariae et S. Georgii de Muntesia, et Bernardum Hugonis de Rupebertinum, castellanum castellaniae Empostae, super baiulia scu praeceptoria locorum de Ulldecona et de La Cenia. Dat. Romae, apud S. Petrum, a. inc. dom. MCDLXXX, XIV kal. dec., pont. a. X. "Humilibus supplicum".

Valencia, A.D.P., Duquesa de Almodóvar, perg. 1083. Ins. in publ. instr. 26 maii 1481. Describitur cum filo can.

SIXTUS (IV) "venerabili fratri nostro Johanni, epo. Albanen., cardinalis Andegaven. ad regnum Franciae et universis Galliarum, provincias ct partes ac illis adiacentia... legato": concedit tibi plurimas facultates super dispensationibus ct privilegiis ut negotia Ecclesiae facilius expedire possis. Dat. Romae, apud S. Petrum, a. inc. dom. MCDLXXXIII, VIII id. oct., pont. a. XIII. "Cum te ad regnum Franciae".

Valencia, A.D.P., Hospital General, perg. 3834. Ins, in pubI, instr. 28 apr. 1487. Membrana parum mutila cum parvis foraminibus et atramenti maculis.

INNOCENTIUS VIII "dilecto filio Johanni de Arevalo, clico., Abulen. dioc., comiti palatini": confirmat ei, suplicatione episcopi Albanen., omnia privilegia concessa per Sixtum IV comitibus palatinis. Dat. Romae, apud S. Petrum, sub anulo piscatoris, die X febr. a. dom. MCDLXXXVI, pont. a. II. "Suplicari nobis humiliter".

Valencia, A.D.P., Hospital General, perg. 3834. Breve. Ins. in publ. instr. 28 apr, 1487. Membrana parum mutila cum parvis foraminibus et atramenti maculis.

ALEXANDER (VI) "dilectis in Christi filiabus nobili mulieri Elionore de Proxida, comitissae Aversae et Mariae de Moncada et de Centelles, ac Ysabele Robert, mulieribus viduis ac dilectis filiis nobili viro Johanni Francisco eciam de Proxida, comiti Almenarae et eiusdem Aversae, ac Graciano Monsoriu, et legum doctori Baltasari de Gallach, laycis Valentin.": indulget eos, ut confessorem eligant, at in tempore interdicti, altarem portatilem habeant. Dat. Romae, apud S. Petrum, a. inc. dom. MCDXCII, VII id. febr., pont. a. I. "Sincerae devotionis affectus" - "voluntatis, indulti et decreti". 
Valencia, A.D.P., Duquesa de Almodóvar, perg. 1293. Cop. not. 26 iun. 1493. Describitur cum filo ser.

Valencia, A.D.P., Duquesa de Almodóvar, e.1.5, caja 1. Cop. not. 9 mart. 1703.

ALEXANDER (VI) "Ad futuram rei memoriam": concedit Francisco de Lonç, fratri Hospitalis S. Iohannis Hierosolimitani, quod, cum aliqua praeceptoria dicti hospitalis sub eadem castellania Empostae existentis vacaret, dicto Francisco de Lonç, juxta formam statutorum et ordinacionum huiusmodi conferri debeat. Dat. Romae, apud S. Petrum, a. inc. dom. MCDXCVII, V id. jan., pont. a. VI. "Soles sedet apostolica" "absolutionis, voluntatis, concessionis, inhibitionis, confirmationis, mandati et derogationis."

Vatencia, A.D.P., Duquesa de Almodóvar, perg. 1073. Cop. not. 6 oct. 1499.

ALEXANDER (VI) "dilecto filio Bernardo Johannis Roig, cler, Valentin.": constituit et assignat Laurentium Barbera, Bernardum Lazer et Franciscum Vinader ad faciendam unam pensionem annuam decem et novem librarum cum dimidia unius librae monetae Valentin., super beneficiis S. Salvatoris, B. Mariae Castellonis de Rugat et super sub Corporis Christi invocationibus in villa de Nijer, dicto Bernardo Iohannis Roig. Dat. Romae, apud S. Petrum, a. inc. dom. MDII, XVII kal. febr., pont. a. XI. "Vitae ac morum honestas" - "absolutionis, reservationis, constitutionis, assignationis, decreti, voluntatis et statuti".

Valencia, A.D.P., Hospital General, perg. 2561. Originale. Bulla dep. Recto, in margine ad s.: $\mathbf{L}$ ad d.: A super plicam ad d.: pro recept. E. Balbes / Io. Denzon sub plica ad s.: ian. et prope taxa: XX (in columna) / A. Draco pro rco / M.Aire? / V. de Phano / P. Tamisan / A. de Palofriis? / And XX (in columna) X ouart Pen. Lettus in parte media: A. Sancto Severino / A. Valdus / G. Alcazar pro (...) ad d.: ? F. Scaput expo(...) A tergo in media pagina: B. Bolis ad s.: A. inc. dom. MCDII die XXVIIl iun. reverendissimus procurator Consilii Asni pens.? A(...) / pro A. de Cugna F. Clementis in margine inf.: S.L. Pugeius? Jo. de Turchis.

Valencia, A.D.P., Hospital General, perg. 2186. Ins. in publ. instr. 8 febr. 1503.

Alexander (VI) "venerabili fratri episcopo Neucastren. et dilectis filiis praeposito eccl. Fresulani ac officiali Valentino": mandat eis, pensionem debitam a Laurentio Barbera, Bernardo Lazer et Francisco Vinader, cler., in favorem Bernardi Johannis Roig, cler. Valentin., integre satisfacere. Dat. Romae, apud S. Petrum, a. inc. dom. MDII, XVII kal. febr., pont. a. XI. "Hodie, dilecto filio".

La Personalidad Internacional de los Papas

Hispania Sacra 48 ( 1996) 
Valencia, A.D.P., Hospital General, perg. 2453. Originale, cum rasis et emendatis in lineis 1 et 4. Bulla dep. Recto in margine ad d.: A in margine sup.: tassa e Nuecastren etiam in reg(...) et pone Neucastren. pro reverendisimo domino vicecancellario Jo. Ragusin? super plicam ad d.: pro receptore E. Balbus / Jo. Denzon sub plica, ad s.: ian. et prope taxa: X / A. Draco pro rco. / M. Aire?/ P. Tamisan/A. de Palofriis? in parte media: A. Sancto Severino/Valdus / G. Alcazar pro (...) A tergo in media pagina: B. Bolis in margine inf.: A. de Sancto Severino P. Filioboni.

Valencia, A.D.P., Hospital General, perg. 2186. Ins. in publ. instr. 8 febr. $J 503$.

IULIUS (II) "Dilecto filio mgco. Johanni Baptiste Brocho, clico. Imolen., scriptori et familiari nostro": facit, constituit et ordenat auctoritatem ei dare ad creandum notarios tabelliones ac iudices ordinarios necnon etiam ad legitimandum naturales bastardos. Dat. Romae, apud [S.] Petrum, a. inc. dom. MDV, VII kal. iul, pont. a. II. "Sincerae devotionis affectus" - "constitutionis, ordinationis, voluntatis, mandati, decreti, concessionis, declarationis, receptionis, agregationis, exemptionis et liberationis".

Valencia, A.D.P., Hospital General, perg. 1417. Ins. in pub]. instr. [.. I sept. 1509. Membrana cum foraminibus in parte media et inf.

LEO (X) "AD PERPETUAM REI MEMORIAM": aprobat et confirmat aedificationem Hospitalis Generalis civitatis Valentin., in loco ubi Hospitalis B. Mariae Innocentium dictae civitatis est, et incorporationem tria aliorum hospitalium pauperum Valentiae: Hospitalis Reginae, S. Lazari et d'En Clapers. Dat. Romae, apud S. Petrum, a. inc. dom, MDXIV, XI kal. iul., pont. a. II. "Altitudo diuinaemprouidentiae" "absolutionis, aprobationis, confirmationis, decreti, suppletionis, unionis, annexionis et incorporationis, indulti, statuti, ordinationis, mandati et voluntatis".

Valencia, A.D.P., Hospital General, perg. 2508. Originale, cum aliquis foraminibus et maculis et cum rasis in lineis 20,23 et 72 . Butla dep. Recto, in margine ad d.: M. P. sub plica ad s. taxa: Gratis de mandato serenissimi domini nostri papae / Jo. Cheminart / Aloisius / C. Wien / G. Gratianis ad d.: P. Bembus / G. Gratianis super plicam ad d.: A. de Castillo A tergo in media pagina: apud me P. Bembum.

Valencia, A.D.P., Hospital General, perg. 2180. Cop. not. 25 ian. 1521. Impressa. Membrana fracta cum magnis foraminibus.

Valencia, A.D.P., Hospital General, perg. 3483. Cop, not. 7 febr. 1545. Describitur cum filo ser.

Valencia, A.D.P., Hospital General, JV-4.2, leg. 11. Cop. simplex s. XVI.

Valencia, A.D.P., Hospital General, IV-4.2, leg. 9. Bulla impressa in chalcographia Josephi Thomae Lucas, qui typographus ab 1736 usque ad 1774 fuit.

La Personalidad Internacional de los Papas Hispania Sacra 48 (1996) 
Translata partim in linguam hispanam: Fragmento de la bula promulgada por León $\mathrm{X}$ sancionando los capítulos vigentes.

LEO (X) "ditectis fíliis decano Corduben. et ministro Sancte Trinitatis extra muros Hispalen. ac priori Sancti Francisci Valentin. monasteriorum": mandat absolutionem Anthonii Cruzado, alias Beteta, oppidi de Ecija, olim ordinis Minimorum, a peccato apostasiae et transitum suum ad ordinem S. Augustini. Dat. Romae, apud S. Petrum, sub anulo piscatoris, die XXVI /... J MDXIX, pont. a. VII. "Exponi nobis".

Valencia, A.D.P., Hospital General, perg. 2095. Breve. Ins. in publ. instr. 14 febr. 1520. Membrana cum margine inf. resecato et cum maculis humiditatis.

CLEMENS VII "venerabili fratri epo. christopolitano Valentiae comoranti et dilectis filiis Ludovico de Perellos ac Hieronymo de Ribelles, canonicis eccliae. Valentin. et eorum cuilibet": committit et mandat absolvere a peccato apostasiae Jacobum Alzina, ordinis heremitarum S. Augustini in domo eiusdem ordinis Barchinon. Dat. Romae, apud S. Petrum, sub anulo piscatoris, die VIII mart. MDXXVI, pont. a. III. "Exponi nobis".

Valencia, A.D.P., Hospital General, perg. 2552. Breve. Ins. in publ. instr. 2 maii 1526. Transcribitur sine loco intra membranam: P. Bombasius, Lact. Caurata.

Paulus III "dilecta in Christo Catherina de Segura, conversa frayla nuncupata ordinis Sancti Bernardi cistercien": absoluit, liberat et dispensat cam ut de ordine S. Bernardi cisterc. ad ordinem canonicorum regularium $S$. Augustini transferri possit. Dat. Tusculi, sub anulo piscatoris, die XXI aug. MDXXXVIII, pont. a. IV. Gratia Dei. "Exponi nobis".

Valencia, A.D.P., Hospital General, perg. 2189. Breve. Ins. in publ. instr. 28 nov. 1538.

PAULUS PP. TERTIUS "AD PERPETUAM REI MEMORIAM"; confirmat, Hospitali Generali civitatis Valentin. omnia et singula privilegia, gratias, concessiones, indulgentias, inmunitates, execuciones, litteras et indulta per predecessores suos con-

La Personalidad Internacional de los Papas

Hispania Sacra 48 (1996) 
cessa. Dat. Romae, apud S. Marcum, sub anulo piscatoris, dic XXVII sept. MDXXXVIII, pont. a. IV. S. Gratia Dei. "Cedis apostolicae benignite".

Valencia, A.D.P., Hospital General, perg. 3483. Breve. Cop. not. 7 febr: 1545.

Gregorius XIII "dilecto filio, officiali Valentino": dispensat Jacobum Yvanes et Raphaelam Çaragoça, ambos civitatis Valentin., ut inter se matrimonium contrahere possint. Dat. Tusculi, a. inc. dom. MDLXXIV, id. apt., pont. a. II. "Oblatae nobis" - "dispensationis".

Valencia, A.D.P., Hospital General, perg. 2185. Originale, membrana mutila in anguio inf. d. et cum aliquis foraminibus. Bulla cum filo can. Recto, in margine ad s.: $L$ ad d.: A super plicam in parte media: J. Lamola ad d.: G. Gaillart sub plica ad s.: ma. et prope taxa: LX/L. Anastasius / P. Balbus, computator / Franciscus Bellotus pro magistris / M. Ruftinus / I. Barengus / Fabri numerus LX ca ad d.: C. Burghesius pro magistro / A. Portigianus / R. Bofidus? A tergo in media pagina: Montagnonus in margine inf.: A. Florienus / P.F. Costa.

Gregorius XIII "Dilecto filio officiali Valentin.": mandat ei provisionem beneficii perpetui ad altarem Sanctissimi Crucifixi, situm in ecclesia Sancte Crucis Valentin., quod vaca: per liberam resignationem Francisci Michaelis de Enquian, in favorem Magini Llobets, presbiteri Tarracon. Dat. Romae, apud S. Petrum, a. inc. dom. MDLXXVIII, id. aug., pont. a. VII. "Dignum arbitramur".

Valencia, A.D.P., Hospital General, perg. 2177. Originale. Bulla cum lilo can. Recio, in margine ad s.: $\mathbf{L}$ in margine ad $\mathrm{d}$.: $A$, super plicam intra foramina bullae: $D$.[Sattelunus] super plicam ad d.: V. Vulcanius sub plica ad s.: sept. et prope taxa: XII (in col.) / B. Girgos / Gi[...] prope: Expta. XVI kal. oct. a. VII / C. Luparus pro magistris in ang. inl. ad s.: L. Anastasius / lo. Pau. Carcanus / Hele numerus XX cui duae lineae superpositae exstant $(=X X I I)$ nus sub plica in parte media: A. Riccius /[A pollegianus] / D. Hatton sub plica ad d.: expedivit duc. tresdecim J. P. Fahonis A tergo, in parte sup.: O. Pamfilius in parte sup. ad d.: R. Betholus in media pagina: Jo. Befel.

Gregorius (XIII) "Dilecto filio offali, Valentin.": concedit Guilielmo Bernardo Catala indultum et suspensionem bonorum assignatorum celebratione anniversarii Arnardi Valleriola in duabus capellaniis, quas ipse in ecclesia S. Catherinae Valentin. construere et aedificare fecit. Dat. Tusculi, a. inc. dom. MDLXXIX, III non. oct., pont. a. VIII. "Inter cetera cordis". 
Valencia, A.D.P., Duquesa de Almodóvar, perg. 436. Originale Bulia dep. Membrana cum una magna macula in parte inf. Recto, super plicam ad d.: J. Brohue sub plica ad s.: oct. et prope taxa: XXX (in col.) / J. Zianus / A. Bergerii / A. de Sto. Petro / L. Conventinus sub plica in ang. inf. ad s.: Vec numerus XXX (in col.) [ess] sub plica in parte media: O. Pamphilius / C. Pamphilius / E. Maronus sub plica ad d.: Io. F. Ruggierius A tergo, in media pagina: Rta. in Secretaria Apostolica. / Jo. F. Ruggierius.

Sixtus (V) "Dilecto filio officiali Valentino": mandat ei, si offic. Dertusen. intra triginta dies Petro Yvanyes, cler. Valentin., beneficium ecclesiasticum in capella B. Mariae virginis in parrochiali ecclesiae Castillionis,Dertusen. dioc., non contulisset et assignavisset, prefatum beneficium dicto Petro Yvanyes conferat et assignet. Dat. Romae, apud S. Petrum, a. inc. dom. MDLXXXVI, XVI kal. febr., pont. a. I. "Hodje dilecto filio".

Valencia, A.D.P., Hospital General, perg. 3833. Originale, cum aliquis foraminibus. Atramentum fere deletum. Bulla dep. Recto ad d.: A super plicam ad d.: Hie. Ghecius sub plica ad. s.: ian. et prope taxa: X / A. Rodriguez / P. Pultrius pro computatore / L. Randonius / B. de Alexandris in parte media: Iu. Barengus / A. Portigianus / A. Lucius A tergo, in media pagina: (Hie. Avila in margine inf. ad s.: Hie. Ma(ffe)us A. Portigianus.

SIXTUS (V), "dilecto filio, officiali Valentin.": dispensat Georgium de Castellvi, dominum baroniae de Carlet, et Annam Margaridam de Calataiut, ut inter se matrimonium contrahere posssint. Dat. Romae, apud S. Petrum, a. inc. dom. MDLXXXVI, VI id. aug., pont. a, II. "Oblatae nobis" _ "dispensationis".

Valencia, A.D.P., Duquesa de Almodovar, perg. 604. Cop. not. 26 aug. 1586. Transcribitur sine loco intra membranam: A. Ponte, a tergo: Rta. A. Justus.

SIXTUS V "AD PERPETUAM REI MEMORIAM": approbat ce confirmat omnia et singula privilegia, indulta, indulgentias... concessa per romanos pontifices predecessores suos Hospitali Generali sub invocatione Apparitionis facta a Domino Nostro Iesuchristo Virgini Maria, olim Sanctorum Innocentium et Sancti Lazari. Dat. Romae, apud S. Petrum, sub anulo piscatoris, die XVII febr. MDLXC, pont. a. V. "Salvator Dominus noster".

La Personalidad Internacional de los Papas

Hispania Sacra 48 (1996) 
Valencia, A.D.P., Hospital General, perg. 3322. Breve. Originale. Atrantentum deletum. Sigillum cereum dep. Recto, infra textun ad d.: M. Vestrius Barbianus A tergo, in ang. sup. ad d.: M. Ants. a Valle.

SIXTUS (V) "AD PERPETUAM REI MEMORIAM": praecipit et mandat, sub pena excommunicationis, notariis et tabellionibus publicis existentibus in Regno Valentiae et civitate Valentin., administratoribus et deputatis Hospitalis Generalis Valentiae copiam authenticam instrumentorum concernentium dicto Hospitali dare et consignare. Dat. Romae, apud S. Petrum, a. inc. dom. MDLXXXIX, XI kal. mart., pont. a. V. "Honestis fidelium" - "absolutionis, precepti, mandatorum et voluntatis".

Valencia, A.D.P., Hospital General, perg. 3342, Originale. Bulla dep. Exstat filum ser. Recto, super plicam ad s.: Auila ad d.: B. Gallus sub plica ad s.: mar. et prope taxa: L / D. de Priotis / A. Vicecancellarius / Builu L rault, Helanus, Pultrius ad $d$.: J. Pirolus / Jo. Genesius $A$ tergo in media pagina: libro primo fol. 23 in margine inf.: Hie[...]or.

Valencia, A.D.P., Hospital General, IV-4.2, leg. 11. Translatun in linguan hispanam.

CLEMENS PAPA VIII "Ad perpetuam rei memoriam": decernet et statuit formam et rationem quae nominibus et institutionibus in rebus erectionis, institutionis, aggregationis, communicationis saecularium archiconfraternitatum et congregationum diversarum nationum, servari debent. Dat. Romae, apud S. Petrum, sub anulo piscatoris, die VII dec. MDCIV, pont. a. XIII. "Quacumque a Sede Apostolica".

Valencia, A.D.P., Hospital General, perg. 2184. Breve. Ins. in publ. instr. 6 iun. 1607. Impressum. Romae, ex typographiae reverendae Camerae Apostolicae MDCVII superiorum permissu.

PAULUS PP. V "Ad perpetuam rei memoriam": concedit aggregationem Confraternitatis Archihospitalis Sancti Spiritus de urbe Saxia et Confraternitatis Archihospitalis Sancti Spiritus Valentiae. Dat. Romae, apud S. Petrum, sub anulo piscatoris, die XIX mart. MDCVII, pont. a. II. "Cum certas confraternitati".

Valencia, A.D.P., Hospital General, perg. 2184. Breve. Ins. in publ. instr. 6 iun. 1607 . Impressum. Romae, ex typographiae reverendae Camerae Apostolicae MDCVII superiorum permissu.

La Personalidad Internacional de los Papas Hispania Sacra 48 (1996) 
Paulus (V) "Dilecto filio officiali Valentin.": mandat ei dispensationem ut matrimonium inter Ludovicum Blasco, militem Militiae Beatae Maria Montesiae et Sancti Georgii, et Franciscam Palau licitum remaneant nihilominus ei secundus consanguinitatis gradus sunt. Dat. Romae, apud S. Marcum, a. inc. dom. MDCX non, aug., pont. a. VI. "Oblatae nobis".

Valencia, A.D.P., Duquesa de Almodóvar, perg. 1137. Transumptum 18 aug. 1610. Transcribitur sine loco intra membranam: C. Bernier.

Paulus V "dilecto filio officiali Dertusensis": mandat ei, si offic. Segobricen, intra triginta dies Hieronimum de Prurto perpetuum beneficiatum in ecclesia S. Martini Valentiae non fecisset, dicto Hieronimo prefatum beneficium conferat et assignet. Dat. Romae, apud S. Marcum, a. inc. dom. MDCXI, VIII kal. mart., pont. a. VII. "Hodie parrochiali ecclesia".

Valencia, A.D.P., Hospital General, perg. 2182. Originale, cum rasis et emendatis in lineis $12,13,18$ et 19 cum aliquis foraminibus et maculis. Bulla cum filo can. Recto, in margine ad d.: A super plicam ad s.: Mor ad d.: A. Danius sub plica ad s.: mar. et prope taxa: XII (in columna) A. Bolodanus? / F. Julianus / F. de Montemayor / V. Costa in parte media: M. Bartolus / Jo. F. Ugolinus / T. a Meyden A tergo ad d.: Avila in margine inf.: P. Mignam...?, Jo. F. Ugolinus.

Paulus (V) "dilecto filio officiali Valentino": mandat ei, ut Petro Pons, rectori ecclesiae parrochialis oppidi de Manuel, parrochialem ecclesiam loci de les Enoves conferat et assignet. Dat. Tusculi, a. inc. dom. MDCXI, VII id. iun., pont. a. VII. "Dignum arbitramur".

Valencia, A.D.P., Hospital General, perg. 2491. Originale, cum aliquis maculis. Bulla dep. Recto, in margine sup. ad. d.: D Z, in margine ad s.: L ad d.: A saper p/ican ad s.: Mor in parte media: $\mathrm{F}$. de Abbatibus ad d.: A. Danius sub plica ad s.: iul. et prope taxa: XVI (in columna) M. Peregrinus / R. Courtinus- Expedita VIl id. iul. a. VIJ / Ant. Carenhus (...)/M. de Magnis? pro pref. solicitt. / F. de Arriaga pro ap. d.s. gr. 2 / J. Bomlielus / Alfumi XXVI (in columna) natus / in parte media: B. de Segnis / Jo. F. Ugolinus / T. a Meyden / Prada pro annata d. 20 et jul. 8 / Hi. Boncompagnus / J. Scalia pro annata d. $104 / \mathrm{J}$. Scalia pro annata. 20 8 ad d.: S. Isquierdus pro annata d $12: 5 / \mathrm{P}$. Miaaldi? / H. Petronius pro annata d $-3 / 2 /$ $\mathrm{Ib}(. .$.$) binus / Expedita d 14 \mathrm{~F}$. de Montemayor $A$ tergo ad $d$. . H. Chausseus / pro magistris in margine inf:: M. Niperesius?, T. a Meyden.

Valencia, A.D.P., Hospital General, perg. 1897 . Ihs. in publ. instr. $20 \mathrm{aug} .161 \mathrm{l}$.

La Personalidad Internacional de los Papas

Hispania Sacra 48 (1996) 
Paulus (V) "dilecto filio officiali Segobricensis": mandat ei, si offic. Valentin. Petro Pons, rectori ecclesiae parrochialis loci de Manucl, parrochialem ecclesiam loci de Les Enoves intra triginta dies non contulisset et assignavisset, dicto Petro Pons, prefatam rectoriam conferat et assignet. Dat. Tusculi, a. inc. dom. MDCXI, VII id, itn., pont. a. VII. "Hodie, cum dilectus filius".

Valencia, A.D.P., Hospital General, perg. 2505. Originale, cum aliquis foraminibus et maculis. Bulla dep. Recto, in margine ad d.: A super plicam ad d.: A. Danius sub plica ad s.: iul. et prope taxa: X M. Peregrinus / R. Courtinus / J. Boraldus? / F. de Arriaga in parte media: B. de Segnis / Jo. F. Ugolinus / T. a Meyden A tergo ad d.: H. Chausseus / pro magistris in margine inf.: M. Bartolus, T. a Meyden.

Paulus (V) "Dilecto filio officiali Valentin.": mandat ei provisionem beneficii ad altarem Francisci, Michaelis et aliorum sanctorum situm in ecclesia Sancti Joannis de Mercato, Valentin. dioc., quod vacat per liberam resignationem Joannis Gabrielis Valenti, in favorem Joannis Baptistae Grau, clerici Valentin. Dat. Tusculi, a. inc. dom. MDCXIII id. maii, pont. a. V. "Dignum arbitramur".

Valencia, A.D.P., Hospital General, perg. 493. Originale. Bulta dep. Recto, in margine ad s.: $\mathrm{L}$ in margine ad d.: A super plicam in parte media: J. Fucciolus super plicam ad d.: A. G. Conde sub plica ad s.: iun. et prope taxa: XII (in col.) / N. de Priotin/J. Bonctus / H. Attorettres pro pref. solvit / Ja. Hie. Miletus / F. de Abbatibus sub plica in ang. inf. ad s.: G. Lom numerus XX cui duae lineae superpositae exstant (= XXII) bau sub plica in partc media: Expedita XII kal.. iul. a. V / Ant. Carenhus pro magistris prope: M. [Gruttis] / C. Pamphilius / V. Carlernes sub plica in ang. inf. ad d.: Expedit duc. 13 D. Canerarius A tergo, in ang. sup. ad d.: V. Carlernes in media pagina: $\mathrm{Rta} . \mathrm{H}$. Chaus[seus] pro magistris.

Paulus (V) "Dilecto filio offrciali Valentin."ö: concedit collationem parrochialis ecclesiae burgi de Cocentaina, Valentin. dioc., Vicentio Rodriguez, clerico dicte dioc., quae per liberam resignationem Simonis Gust vacat. Dat. Romae, apud S. Mariam Maiorem, a. inc. dom. MDCXIII id. oct., pont. a. IX. "Dignum arbitramur".

Valencia, A.D.P., Hospital General, perg. 3832. Originale. Bulla dep. Membrana mulila in parte sup. Recto, in margine inf. ad s.: $\mathrm{L}$ in margine ad d.: A super plicam in parte media J. B[ar]tus super plicam ad d.: L. de Sancey sub plica ad s.: oct. et prope taxa: XX (= in col. $)$ / B. Sena pro .../M. Bergerettus / P. Caval prope: Expedita $4 \mathrm{kal}$. nov. a. IX / H. Gillon pro magistris sub plica in angulo inf. ad s.: P. J. Beneltus / D. Canevarius / Asti numerus XX (= in col.) us sub plica in parte media: B. de Segnis / Jo. F. Ugolinus / T. a Meyden sub plica in ang. inf. ad 
d.: expedit ... 13 P. J. Beneltus A tergo, in parte sup.: B. de Segnis / L. Ursinus Uniarius in parte sup. ad d.: T. a Meyden in media pagina: M. Maur[...] pro magistris.

Urbanus VIII "Dilecto filio officiali venerabilis fratris nostri archiep. Valentin.": mandat ei collationem preceptoriae Sancti Antonit prope et extra muros Valentin. , quae primo per obitum Raymundi Oliva, seu Olivares, et postquam Jacobi Salt vacat, in favorem Lucae Gilbau, canonici ordinis S. Antonii Viennen, sub regula S. Augustini, Dat. in orte Gandulfi, dioc. Albanen., a. inc. dom. MDCXXXVI XIII kal, maii, pont. a. XIII. "Dignum arbitramur".

Valencia, A.D.P., Hospital General, perg. 13. Originale. Membrana cum foraminibus et rasis in fere omnibus lin. Bulla cum filo can. Recto, super plicam in ang. sup. ad s.: $f$. v. super plicam ad d.: L. Radeux sub plica ad s.: oct. et prope taxa: XXX ( $=$ in col.) / N. Gottus pro ... / V. [Berme] / Herl... pro prefecto / M. Guonis / J. Casalis / H. Fernadon capellanus sub plica in ang. inf. ad s.: O numerus XXX (=in col.) dan prope: V cum una linea superposita, prope: Expedita V kal. nov. a. XIIl / C. Simonellus pro magistris sub plica intra foramina bullae: A. Perelius? / S. Ugolinus / N. Filipundius A tergo, in ang. sup. ad s.: N. Filipundius in media pagina: ... in parte inf. ad s.: a. inc. dom. MDCXXXVI die XXII apr. registratus Guillielmus Mauritius propris bonioni et letrarum expeditioni confensit/ Francus. Rocolinus?

ALEXANDER VII "AD FUTURAM REI MEMORIAM"ö: assignat suplicatione Philippi, Hispaniarum regis, Hospitali Regali et Generali civitatis Valentin. pensionem annuam ad viginti annos duarum millium librarum super fructibus, redditibus et proventibus mensae archiepiscopalis Valentin. Dat. Romae, apud S. Petrum, a. inc. dom. MDCLV XIV kal. maii, pont. a. I. "Rationi congruit" - "nominationis, voluntatis, decreti et mandati".

Valencia, A.D.P., Hospital General, perg. 3482. Originale. Bulla cum filo ser. Recto, super plicam ad s.: Rig.: 20 super plicam ad d.: visa Hionequius / Johan Baurus sub plica ad s. taxa: LXXXVIIII (=in col.) / maii prope: L. Radeux prope Terta / D. de Orchis / D. Crobis? pro annata solvit in provisione / Huibruesus capellanus sub plica in ang. inf. ad s.: De La numerus LXXXXVIIII (= in col.) Borne sub plica in parte media: Palmerius pro annata solvit in provisione / Womes? capellanus / P. Thedenhus pro annata solvit in provisione / P. B. Pheus sub plica ad d.: G. Carbo / Jo. Espinosa / M. Verospinus pro annata solvit in provisione / Hionequinus capellanus / Calorus pro annata solvit in provisione / Jo. de Sennicolis capellanus A tergo, in media pagina: Rta, in Camera Apostolica.

La Personalidad Internacional de los Papas

Hispania Sacra 48 (1996) 
Alexander (VII) "Dilectis filiis magistro Marco Antonio Tomado in utraque signatura nostra referendario, ac antiquiori canonico ecclesiae Valentin., et officiali venerabilis fratris nri. archiepiscopi Valentin.": mandat eis pensionem Hospitali Generali civitatis Valentin. super fructibus, redditibus et proventibus mensae episcopalis Oriolen. integre persolvi. Dat. Romae, apud S. Mariam Maiorem, a. inc. dom. MDCLVIII id. mart., pont. a. IV. "Hodie Hospitali Generali" - "interdicti et suspensionis".

Valencia, A.D.P., Hospital General, IV-4.2/ leg. 11. Cop, not. s. XVII. Transcribitur sine loco intra membranam: ian. X/S. Gasparrus / J. Agnellus / J. Marliis / J. A. Amitus card.

INNOCENTIUS PP. XII "Dilecto filio officiali Valentin.": committit et mandat ei dispensationem matrimonio inter Josephum de Cardona y Pertusa et Rosam de Cardona Vivas de Cariamas, quae in secundo consanguinitatis vel affinitatis gradu sunt. Dat. Romae, apud S. Mariam Maiorem, sub anulo piscatoris, die V aug. MDCXCVII, pont. a. VII. "Oblata nobis".

Valencia, A.D.P., Duquesa de Almodóvar, perg. 431. Breve. Originale. Sigillum cereum. Recto, infra textum ad d.: B. card. Paodas A tergo, in ang. sup. ad d.: A. de Laurentiis.

INNOCENTIUS XIII "AD FUTURAM REI MEMORIAM": assignat suplicationem Philippi, Hispaniarum regis, Hospitale Regale et Generale civitatis Valentin. pensionem annuam ad quattuordecim annos ducentorum quadraginta ducatorum auri de camera sexdecim iuliorum super fructibus, redditibus et proventibus mensae episcopalis Oriolen. Dat. Romae, apud S. Petrum, a. inc. dom. MDCCXXI XV kal. iun., pont. a. I. "Rationi congruit".

Valencia, A.D.P., Hospital General, perg. 3054. Originale. Atramentum in parte media deletum. Bulla cum filo ser. Recto, super plicam in ang. sup. ad s.: Idem prope XXXX (=in col.) et $X$ in parte media: Rio: 9 ? super plicam ad d.: sdr / visa pro R. P. D. Sinibaldo / H. Palucelius... / C. Vattus sub plica ad s.: mar, et prope taxa: XXXX (=in col.) / H. Rota / F. Valentin / C. [Llanus] / pro annata solvit in manibus hic colectoris generalis / ... Mopuccius? / F. de Feluchis / J. Je numeri XXXX (=in col) et X hoÿe prope A. Mastronius sub plica ad d.: P. card. And. / R. Pebruccies ... / pro annata solvit in manibus sub ... / J. A. Sernicoli... A tergo, in media pagina: Rta. in Camera Apostolica / A. Pelucianus?. 
Innocentius XIII "dilectis filiis canonicorum curiae Camerac Apostolicae, generali auditori ac venerabilium fratrum nostrorum archiepi. Valentin. et epi. Segobricen. officialibus": assignat Hospitale Regale et Generale Valentin. pensionem annuam ad quattuordecim annos ducentorum quadraginta ducatorum auri de canera sexdecim iuliorum super mensae episcopalis Oriolen, fructibus, redditibus et proventibus. Dat. Romae, apud S. Petrum, a. inc. dom. MDCCXXI XV kal. iun., pont. a. I. "Dudum felicis recordationis".

Valencia, A.D.P., Hospital General, perg. 3831, Originale. Bulla cum filo can, Recto, super plicam in ang. sup. ad s.: Idem super plican ad d.: C. Vattus / visa pro R. P. D. Sinibaldo / H. Palucelius... sub plica ad s. taxa: X/H. Rota / F. Valentin. A tergo, ad s.: Rta. in Camera Apostolica A. Pelucianus?

Benedictus (XIII) "dilectis filiis canonicotum curiae Camerae Apostolicae, generali auditori ac venerabilium fratrum nostrorum Oriolen. et Segobricen. episcoporum of ficialibus": assignat Hospitale Regio et Generali civitatis Valentin. pensionem annuam ad quattuordecim annos noningentorum quadraginta unius ducatorum auri de camera et trium iuliorum super mensae archiepiscopalis Valentin. fructibus, redditibus et proventibus. Dat. Romae, apud S. Petrum, a. inc. dom. MDCCXXV XIV kal. maii, pont. a. I. "Hodie Regio et Generali Hospitali".

Valencia, A.D.P., Hospital General, perg. 3831. Originale. Bulla dep. Recto, super plicam in ang. sup. ad s.: Idem super plicam ad d.: C. Vattus / visa pro R. P. D. Sinibaldo / H. Palucelius ... sub plica ad s. taxa: X / F. Valentin / Iu. Rentrou prope: Francus. Massanius ... A tergo ad s.: Rta. in Camera Apostolica / A. Pierunius.

BENEDICTUS (XIII) "dilecto filio Petro Plaza, comendatori domus et Hospitalis S. Antonii Abbatis Valentin.": conferit et providit ei commenda S. Antonii Abbatis Valentin. Dat. Romae, apud S. Petrum, a. inc. dom. MDCCXXIX, IV non. ian., pont, a. VI. "Religionis zelus virtutum".

Valencia, A.D.P., Hospital General, perg. 3422. Originale, cum aliquis maculis. Membrana mutila in angulis inf. Bulla dep. Recto, super plicam ad s.: M(asiu)s in parte media A g n / Enlla? ad d.: (...ulo...) sub plica ad s.: L et in parte media: $C$ sub plica ad s.: ian. et prope taxa: XX (in columna) / Jam? de Camonys? / J.B Canceius? / Franciscus Massanius (...) / J. de Feliich, capellanus / A. Gior XXX (in columna) gattus V. Billanelius / Expedita id. ian. a. VI / R. de Abbatibus pro magistris in parte media: I. Caballinus / N.? Soderinus / A. Caraffa, cardinalis A tergo ad d.: Lucerius?

La Personalidad Internacional de los Papas

Hispania Sacra 48 (1996) 
CLEMENS PP. XII "AD FUTURAM REI MEMORIAM": prorogat, extendit et ampliat Hospitali Generali civitatis Valentin. pensionem annuam noningentorum quadraginta unius ducatorum auri et trium iuliorum monetae romanae super fructibus, redditibus et proventibus mensae archiepisc. Valentin. Dat. Romae, apud S. Mariam Maiorem, sub anulo piscatoris, die XXIII apr. MDCCXXXIX, pont. a. IX. "Exponi nobis".

Valencia, A.D.P., Hospital General, perg. 3532. Originale. Breve. Sigillum dep. Recto, infra textum ad d.: Reverendus cardinalis Passioneus.

BENEDICTUS PP. XIV "AD FUTURAM REI MEMORIAM": extendit et prorogat ad alios quattuordecim annos pensionem annuam noningentorum quadraginta unius ducatorum auri de camera et trium juliorum super fructibus, redditibus et proventibus mensae archiepiscopalis Valentin. Hospitali Regio et Generali civitatis Valentin. Dat. Romae, apud S. Mariam Maiorem, sub anulo piscatoris, die IX iul. MDCCLIII, pont. a. XIII. "Alias felices recordationes" - "prorogationis".

Valencia, A. D. P., Hospital General, perg, 3531. Breve. Originale. Sigillum ceretun. Recto, infra textum ad s.: Registrata in libro Diversorio curiae / ecclesiasticae Valentinae, de anno millesimo septingentesimo quinquagesimo ter/tio die I decembris in Archivio dictae / curiae recondito / Itta est / Guillermus Aparicio pro / archivero infra textum ad d.: Pro domino cardinali Passioneo / Joannes Florius substitutus.

CLEMENS PP. XIII "AD FUTURAM REI MEMORIAM": prorogat, per alios quattuordecim annos, unam pensionem annuam ducentorum quadraginta ducatorum de camera et sexdecim iuliorum super fructibus, redditibus et proventibus mensae episcopalis Oriolen. Hospitali Regali et Generali civitatis Valentin. Dat. Romae, apud S. Mariam Maiorem, sub anulo piscatoris, die VII maii MDCCLX, pont. a. П. "Exponi nobis".

Valencia, A.D.P., Hospital General, perg. 3533. Breve. Originale. Sigillum cereun. Recto, infra textum ad s.: Registrata in libro Diversorio curiae / ecclesiasticae Valentinae anni millesimi septingentesimi sexagesimi primi sub die vigesimo primo ianuarii, in eitısdem ar/chivio recondito / Itta est / Guillermus Aparicio ... / pro Archivero infra textun ad d.: pro domino cardinali Passioneo / Joannes Florius substitutus.

Vajencia, A.D.P., Hospital General, IV-4.2, leg. 11. Cop. simplex 1763.

La Personalidad Internacional de los Papas Hispania Sacri 48 (1996) 
PIUS (VI) "AD FUTURAM REI MEMORIAM": constituit Hospitali Generali civitatis Valentin. pensionem annuam quingentorum nonaginta duorum ducatorum auri monetae romanae super fructibus, redditibus et proventibus mensae archiepisc. Valentin. ad quattuordecim annos. Dat. Romae, apud S. Petrum, a. inc. dom. MDCCLXXIV, VIII kal. mart., pont. a. I. "Rationi congruit" _ "absolutionis, nominationis, voluntatis et decreti".

Valencia, A.D.P., Hospital General, perg. 3829. Originale. Bulla dep. Exstat filun ser. Recto, super plicam, ad s.: P.L. dattarius Pd 26: 4 ad d.: Ph. Martinellus sub plica ad s.: mar. et prope taxa: XXXXVII (in columna) / I. Pediconi / C. Battaglia- M. Antognettus / A. C. Angelini A. / Pro communi soluit in manibus magistri / F. Moffuceus? / A. Puniatti? capellanus gad. / A. Be XXXXVII (in columna) nucei J. Sxuze? in parte media: Pro communi soluit in manibus domini subsecretarii generalis Pro communi soluit in manibus domini subsecretarii generalis / R. Mathoni pro capeltano / Pro communi soluit in manibus domini subsecretarii generalis / Franciscus Fontana, capellanus ad d.: Pro communi soluit in manibus domini subsecretarii generalis / F. de Turre, secretarius / Pro communi soluit in manibus domini subsecretarii generalis / Antonius Sernicoli, secretarius / Pro communi soluit in manibus meis pro Sacro Collegio / Sanctae Mariae nonaginta XX et (...) $40=$ exstat / A Cans? de Turre, subsecretarius generalis $A$ tergo, in media pagina: Podin in margine inf.: (...).

Valencia, A.D.P., Hospital General, IV. 4. $1 / 2$ ff. 3 r. - 5 v. Cop. in bullario 1781.

Pius (VI) "dilectis filiis magistro Nicolao Riganti, in utraque signatura nostra referendario ac antiquiori canonico ecclesiae Valentin. et officiali ven(erabili) fratris nostri archiepiscopi Valentini": mandat eis pensionem perpetuam quingentorum nonaginta duorum ducatorum auri super fructibus, redditibus et proventibus mensae archiepiscopalis Valentin. Hospitali Generali civitatis Valentiae facere. Dat. Romae, apud S. Petrum, a. inc. dom. MDCCLXXIV, VIII kal, mart., pont. a. I. "Dudum felicis recordationis".

Valencia, A.D.P., Hospital General, perg. 3828. Originale. Bulla dep. Recto, super plicam ad s.: P.L. dattarius ad d.: Ph. Martinellus sub plica ad s. taxa: X / I. Pediconi / C. Battaglia / F. Moffuceus / A. Puniati? capellanus gad. A tergo: L. Podin.

Valencia, A.D.P., Hospital General, IV- 4.1/2, ff, 5 v. -6 v. Cop. in bullario 778 I.

PIUS PP. VI "AD FUTURAM REI MEMORIAM": extendit et prorogat ad alios quattuordecim annos Hospitali Regali et Generali civitatis Valentin., pensionem annuam ducentorum quadraginta ducatorum auri de camera, et sexdecim iuliorum monetae romanae super fructibus, redditibus et proventibus mensae episcopalis Oriolen..

La Personalidad Internacional de los Papas

Hispanin Sacra 48 (1996) 
Dat. Romae, apud S. Mariam Maiorem, sub anulo piscatoris, die IV aug. MDCCLXXV, pont. a. I. "Exponi nobis".

Valencia, A.D.P., Hospital General, perg. 3526. Originale. Breve. Sigillum dep. Recto, infra textum ad s.: Iudex cardinalis de Comitibus.

Valencia, A.D.P., Hospital General, IV-4.1/2, ff. 1 r. - 2 v. Cop. in bullario 1781 .

Pius papa VI "Ad futuram rei memoriam": prorogat et extendit alios quattuordecim annos pensionem annuam noningentorum quadraginta unius ducatorum auri de camera et trium iuliorum super fructibus, redditibus et proventibus mensae archiepiscopalis Valentin. Regio et Generali Hospitali civitatis Valentin. Dat. Romae, apud S. Petrum, sub anulo piscatoris, die XXV iun. MDCCLXXXI, pont, a. VII. "Alias nobis expositum".

Valencia, A.D.P., Hospital General, IV-4.1/2, ff. 7 r. - 8 v. Breve. Cop. in bullario 1781. Transcribitur sine loco intra membranam: Iudex cardinalis de Comitibus / Registrado fol. 1624.

PIUS PP. VI "AD FUTURAM REI MEMORIAM": prorogat extendit et ampliat ad alios quattuordecim annos pensionem annuam quingentorum nonaginta duorum ducatorum auri de camera super fructibus, redditibus et proventibus mensae archiepiscopalis Valentin. Dat. Romae, apud S. Petrum, sub anulo piscatoris, die XXVIII iun. MDCCXCI, pont. a. XVII. "Exponi nobis".

Valencia, A.D.P., Hospital General, perg. 3527. Breve. Originale. Sigillum cereun. Recto, infra textun ad s.: Registrado fol. 2605 infra textum ad d.: Reverendus cardinalis Braschius de Honestis.

Valencia, A.D.P., Hospital General, IV-4. /2, ff. 12 r, - 13 v. Cop. in bullario 1791.

Pius PP. VI "Ad futuram rei memoriam": extendit et prorogat ad quattuordecim annos unam pensionem annuam ducentorum quadraginta ducatorum auri de camera et sexdecin iuliorum super fructibus, redditibus et proventibus mensae episcopalis Oriolen. Dat. Romae, apud S. Petrum, sub anulo piscatoris, die XXVIII iun. MDCCXCI, pont. a. VII. "Nuper pro parte".

Valencia, A.D.P. Hospital General, IV-4.1/2, ff. 9 r. - 11 v. Breve. Cop. in bullario 1791. Transcribitur sine loco intra membranam: Reverendus cardinalis Braschius de Honestis / Registrado fol. 2605 .

La Personajidad Internacional de los Papas Hispania Sacra 48 (1996) 
PIUS PP. VII "AD FUTURAM REI MEMORIAM": prorogat et extendit ad quattuordecim annos, Hospitali Generali civitatis Valentin., unam pensionem annuam noningentorum quadraginta unius ducatorum auri de camera et iutiorum trium monetae romanae super fructibus, redditibus ac proventibus mensae archiepiscopalis Valentin. Dat. Romae, apud S. Mariam Maiorem, sub anulo piscatoris, die XXIII ian. MDCCCI, pont. a. I. "Expositum nobis".

Valencia, A.D.P., Hospital General, perg. 3528. Origincte. Breve. Sigillin ceretun. Recto, infra textum ad d.: Reverendus cardinalis Braschius de Honestis.

Valencia, A.D.P., Hospital General, IV- 4. 1/2, f. 14 r. - 15 v. Cop. in bullario 1801.

PIUS PP. VII "AD FUTURAM REI MEMORIAM": prorogat, extendit et ampliat ad alios quattuordecim annos pensionem annuam quingentorum nonaginta duorum ducatorum auri de camera super fructibus, redditibus ac proventibus mensae archiepiscopalis Valentin. Hospitali Regio Generali civitatis Valentin. Dat. Romae, apud S. Mariam Maiorem, sub anulo piscatoris, die III iun. MDCCCVI, pont. a. VII., "Nuper nobis exponi" - "prorogationis".

Valencia, A.D.P., Hospital General, perg. 3529. Breve. Originale. Sigillum cereun. Recto infra textun ad d.: Reverendus cardinalis Braschius de Honestis.

Valencia, A.D.P., Hospital General, IV-4.1/2, ff. 16 r. -17 v. Cop. in bullario /806.

PIUS VII "AD FUTURAM REI MEMORIAM": prorogat, extendit et ampliat ad quattuordecim annos pensionem annuam ducentorum quadraginta ducatorum auri de camera et sexdecim iuliorum monetae romanae super fructibus, redditibus et proventibus mensae episcopalis Oriolen., Hospitali Generali Valentiae. Dat. Romae, apud S. Mariam Maiorem, sub anulo piscatoris, die III iun. MDCCCVI, pont. a. VII. "Expositum nobis".

Valencia, A.D.P., Hospital General, perg. 3530. Originale. Breve. Sigillum cereum. Recto, infra textum ad d.: Reverendus cardinalis Braschius de Honestis.

Valencia, A.D.P., Hospital General, IV-4.1/2, ff. 18 r. - 20 r. Cop. in bullario $/ 806$.

La Personalidad Internacional de los Papas

Hispania Sacra 48 (1996) 
PIUS (VII) "AD FUTURAM REI MEMORIAM": constituit Hospitali Regali et Generali civitatis Valentin., pensionem annuam ducentorum viginti et unjus ducatorum auri de camera et septem iuliorum monetae romanae, super fructibus, redditibus et proventibus mensae episcopalis Oriolen., ad quattuordecim annos. Dat. Romae, apud S. Mariam Maiorem, a. inc. dom. MDCCCXV, XV kal. jan., pont. a. XVI. "Ex debito pastoralis" ( "absolutionis, reservationis, constitutionis, assignationis, decreti, mandati, statuti et voluntatis".

Valencia, A.D.P., Hospital General, perg., 3464. Originale, cum rasis et emendatis in lineis 37 et 38 . Bulla cum filo ser. Recto, super plicam ad s.: P? demus? ad d.: Ph Matthe(...) sub plica ad s. taxa: Nov. XXXXVIt (in columna) N. Grappelil / N. Minor / Ph. Antognettu / Pro communi soluit in manibus domini subsecretarii generalis / Mincia, capellanus / Pro B. Ad. / C. Benettini? subsecretarius / J. Battaglia, capellanus / J. MartiXXXXVIInellus G. Egidi in parte media: Aljaja? - Pro communi soluit in manibus domini subsecretarii generalis / Pro communi soluit in manibus domini subsecretarii generalis / Iohannes Sernicoli pro capellano / Pro communi soluit in manibus domini subsecretarii generalis / $\mathrm{Ph}$. Camapei, secretarius ad d.: LC / Pro communi soluit in manibus domini subsecretarii generalis / Ph. Camapei pro capellano / Pro communi soluit in manibus meis / scuta 8te 13: et oboli 70 etiam/ pro Sacro Collegio / Iohannes Sernicoli subsecretarius A tergo in media pagina: L. Podin.

PIUS (VII) "AD FUTURAM REI MEMORIAM": reservat, constituit et assignat Hospitali Regali et Generali civitatis Valentin., pensionem annuam quadringentorum septuaginta ducatorum auri de camera et decem iuliorum monetae romanae, super fructibus, redditibus et proventibus mensae episcopalis Segobricen., ad quattuordecim annos. Dat. Romae, apud S. Mariam Maiorem, a. inc. dom. MDCCCXVI, VII kal. apr., pont. a. XVII. "Ex debito pastoralis" ( "absolutionis, reservationis, constitutionis, assignationis, decreti, mandati, statuti et voluntatis".

Valencia, A.D.P., Hospital General, perg. 3463. Originale, cum maculis, cum rasis et emandatis in linea 55. Buila cum filo ser. Recto, super plicam ad s.: P? deus? F R 1:7 LIII (tres lineolae in linea) ad d.: Ph. Mattho(...) 8 P 3 sub plica ad s.: mar. et prope taxa: LIII / F. Aretucci / I. Snatti? / Expedita VII kal. apr, a. XVIl / Ph. Antognetus / Pro communi soluit in manibus subsecretarii generalis / Mincia Cappnus / Pro? B. Ad. / C. Benettini, subsecretarius / J. Battaglia pro capeltanus / V. Do LIIl (tres lineolae in linea) nati, G. Egidi in parte media: C Aliaja? / G. A. Belletti(...) / Pro communi soluit in manibus domini subsecretarii generalis / Pro communi soluit in manibus domini subsecretarii generalis / lohannes Sernicoli pro capellano / Pro communi soluit in manibus domini subsecretarii generalis / D. Grappelii secretarius / Pro communi soluit in mantbus domini subsecretarii generalis / lohannes Sernicoli, secretarius / Pro communi soluit in manibus domini subsecretarii generalis / Ph. Camapei, secretarius / Pro communi soluit in manibus domini subsecretarii generalis / scuta (...) sexaginta octo et ob. 75 etiam pro / Sacro Colfegio et(...) / Raphael Matheum, subsecretarius ad d.: L.C. 
Valencia, A.D.P., Hospital General, IV-4.1/2, ff. $20 \mathrm{v},-25$ r. Cop. in bullario 1816 .

Valencia, A.D.P., Hospital General, IV-4.2, leg. 11. Transumptum et transiatum 27 mart. 1816.

Leo (XII) "Ad futuram rei memoriam": reservat de novo pensionem Xenodochii Generalis Valentin. super fructibus, redditibus et proventibus mensae episcopalis Oriolen, mille ducentorum et trium ducatorum auri et duorum iuliorum, subrogatam et translatam ad mensam episcopalis Malacitan. Dat. Romae, apud S. Petrum, a. inc. dom. MDCCCXXVI VII kal. sept., pont. a. II. "Ex apostolicae potestatis" - "voluntatis, statuti, mandati et derogationis".

Valencia, A.D.P., Hospital General, IV-4.I/2, ff. 25 r. - 29 v. Cop. in bullario 1826.

La Personalidad Internacional de tos Papas

Hispania Sacra 48 (1996) 


\section{ÍNDICES}

\section{INDEX "DE INCIPIT"}

doc. $n$.

doc. $\mathrm{n}$.

$\begin{array}{lr}\text { Alias felices recordationes } & 56 \\ \text { Alias nobis expositum } & 61 \\ \text { Altitudo divinae providentiae } & 27 \\ \text { Aut movet nos } & 18 \\ \text { Cedis apostolicae benignite } & 31 \\ \text { Cum certas confraternitati } & 40 \\ \text { Cum te ad regnum Franciae } & 20 \\ \text { Dignum arbitramur } 15 ; 33 ; 43 ; 45 ; 46 ; 47 \\ \text { Dudum felicis recordationis } & 52 ; 59 \\ \text { Dum ad illam } & 11 \\ \text { Et si ex debito } & 17 \\ \text { Ex apostolicae potestatis } & 69 \\ \text { Ex debito pastoralis } & 67 ; 68 \\ \text { Ex iniuncto nobis } & 2 \\ \text { Exponi nobis 28; } 29 ; 30 ; 55 ; 57 ; 60 ; 62 \\ \text { Expositum nobis } & 64 ; 66 \\ \text { Hodie cum dilectus filius } & 44 \\ \text { Hodie dilecto filio } & 25 ; 35 \\ \text { Hodie Hospitali Generali } & 49 \\ \text { Hodie parrochiali ecclesia } & 42 \\ \text { Hodie Regio et Generali Hospitali } & 53\end{array}$

Honestis fidelium

Humilibus supplicum 19

Inter cetera cordis $\quad 34$

Illius qui a Patre 12

Litterarum scientia 6

Nuper nobis exponi 65

Nuper pro parte $\quad 63$

Oblata nobis $\quad 50$

Oblatae nobis $\quad 10 ; 32 ; 36 ; 41$

Pia fidelium vota 8

Provenit ex nostrae devotionis 4

Quacumque a sede apostolica $\quad 39$

Quoniam, ut ait apostolus 3;7

Rationi congruit $\quad 48 ; 51 ; 58$

Religionis zelus virtutum $\quad 54$

Sedis apostolicae providentia $\quad 13$

Sincerae devotionis affectus $1 ; 22 ; 26$

Soles sedet apostolica 23

Solet nimirum Romani Pontifices 16

Suplicari nobis humiliter 21

Vitae ac morum honestas $5 ; 9 ; 14 ; 24$

\section{INDEX LOCORUM ET NOMINUM ${ }^{18}$}

Albanensis, ep., 20, 21

Alexander pp. VI, 22, 23, 24, 25

Alexander pp. VII, 48, 49

Alphonsus V, rex Aragonum, 12

Alzina, Jacobus, ord. her. S. Augustini, 29

Amedeus, dux Sabaudiae, 12

Andegavensis, card., 20

Andreae, Bernardus, 3

18 Nomina personarum et locorum servando documentorum graphiam transcripta sunt. 
Arevalo, Johannes de, cler. Abulen dioc., 21

Armenguer, Johannes, 3,4

Auditor Generalis, 52, 53

Barbera, Laurentius, cler., 24, 25

Barcelo, Franciscus, cler. Valentin., 3, 4

Barchinona, Barchinonensis

Domus S. Augustini, 29

Offic., 18

B. Maria Castellonis de Rugat, eccl., Valentin. dioc., 24

B. Maria Magdalena Caesaraugustae, eccl. parroch., 6

B. Maria et S. Georgius Montesiae, magister militiae, 19, 41

Benedictus pp. XIII (Petrus de Luna), 1, 2, 3, 4, 5, 6, 7, 8, 9, 10

Benedictus pp. XIII, 53, 54

Benedictus pp. XIV, 56

Blasco, Ludovicus, miles Militiae B: Mariae et S. Georgii Montesiae, 41

Bovia, Petrus de, 3

Brocho, Johannes Baptista, cler. Imolen. dioc., 26

Cabrera, Franciscus, 15

Cesaraugusta, 6,11

Calataiut, Anna Margarida de, 36

Calbo, Sancius, 3,4

Calistus pp. III, 17

Cardona Vivas de Cañamas, Rosa de, 50

Cardona y Pertusa, Joseph de, 50

Castello de Rugat, vid. B. Maria Castellonis de Rugat, ecel.

Castellvi, Georgius de, dominus baroniae de Carlet, 36

Castillio, Dertusen. dioc., eccl. paroch, 35

Catala, Guilielmus Bernardus, 34

Catala, Bernardus, 10

Cathalonia, principatus, 18

Centelles, Johanneta de, 10

Clavell, Berengarius, can. Valentin., 19

Clavell, Petrus, vic. eccl. partoch. B. Mariae Caesaraugustae, 6

Clemens pp. VII, 29

Clemens pp. VIII, 39,

Clemens pp. XII, 55

Clemens pp. XIII, 57

Cocentaina, Val. dioc., eccl. parroch., 46

Compayn, Johannes, pres. ben. in eccl. parroch. S. Michaelis de Cardona, Urgellen. dioc., 5

La Personalidad Internacional de los Papas

Hispania Sacra 48 (1996) 
Confraternitas Archihospitalis S. Spiritus in Saxia, 40

Confraternitas Archihospitalis S. Spiritus Valentiae, 40

Cordubensis, dec., 28

Cruililes, Franciscus, can. Valentin., 13

Cruzado, Anthonius (alias Beteta), ord. fratrum minimorum, 28

Curia Camerae Apostolicae, can., 52, 53

Çaplana, Petrus, 3, 4

Çaragoça, Raphaela, 32

Dertusensis, offic., 9, 18, 35, 42

Dezpuig, Ludovicus magister militiae B. Mariae et S. Georgii de Muntesia, 19

Dominguez, Jacobus, 3, 4

Ecija, Hisp. dioc., 28

Enguera, Val. dioc. praeceptoria, 16

Enquian, Franciscus Michaelis, 33

Eugenius pp. IV, 11, 12, 13

Francia, regnum, 20

Fresulanis, praep., 25

Gallach, Baltasar de, legum doctor, 22

Galliae, 20

Garciae, Ferdinandus, 3, 4

Gilbau, Luca, can. ord. S. Antoni Viennen., 47

Grau, Johannes Baptista, cler. Valentin., 45

Gregorius pp. XIII, 32, 33, 34

Gust, Simon, 46

Hospitalis B. Mariae de Gracia Caesaraugustae, 11

Hospitalis d'En Clapers Valentiae, 27

Hospitalis Generalis Valentiae, $27,31,37,38,48,49,51,52,53,54,55,56,57,58$,

$59,60,61,62,63,64,65,66,67,68,69$

Administratores, 38

Hospitalis Reginae Valentiae, 27

Hospitalis S. Antoni Abbatis Valentiae, 54,

Hospitalis S. Johannis Hierosolimitani, 23

Hospitalis S. Lazari Valentiae, 27, 37

Hospitalis SS. Innocentium Valentiae, I, 2, 3, 4, 8, 15, 17, 27, 37

Administratores, 1, 2,8

Conditores, 4

Hospitalarius, 4

Imolensis dioc., vid. Brocho, Johannes de

Innocentius, pp. VIII, 21

Innocentius, pp. XII, 50 
Innocentius, pp. XIII, 51

Ixar, Gundisalvus de, 16,

Iulius pp. II, 26

Johannes, ep. Albanen., 20

La Çaydia, mon. ord. prope muros Valentiae, ord. cisterc., vid. S. Bernardus, mon.

extra muros Valentiae, ord. cisterc.

La Cenia, Dertusen. dioc., baiulia seu praeceptoria, 19

Lazer, Bernardus, cler. Valentin., 24, 25

Leo pp. X, 27, 28

Leo pp. XII, 69

Les Énoves, Valentin. dioc., eccl. parroch., 43, 44

Lonç, Franciscus de, frater Hospitalis S. Johannis Hierosolimitani, 23

Llobets, Maginus, pres. Tarraconen., 33

Malacitan., mensa ep., 69

Maldano, Tarraconen. dioc., eccl. parroch., 5

Manso, Aduren. dioc., vid. S. Quiteria, mon. ord. S. Benedicti

Manuel, Valentin. dioc., eccl. parroch., 43, 44

Moncada et de Centelles, Maria de, 22

Monsoriu, Gracianus, 22

Montisalbanus, praeceptoria, Caesaraugusten. dioc., 16

Muntesia, militia B. Mariae et S. Georgii de Muntesia, 19, 41,

Museros, Valentin. dioc., 16

Neucastrensis, ep., 25

Nicolaus pp. V, 14, 15, 16

Nijer $^{19}, 24$

Oliva (seu Olivares), Raymundus, 47

Oriolensis

Ep., 53

Mensa ep., $49,51,52,57,60,63,66,67,69$

Offic., 53

Orthetha, Valentin. dioc., praeceptoria, 16

Palau, Francisca, 41

Paulus pp. III, 30, 31

Paulus pp. V, 40, 41, 42, 43, 44, 45, 46

Pedrea, Petrus, 3, 4

Perellos, Ludovicus de, can. Valentin., 29

Philippus, Hispaniarum rex, 48, 51

Pius pp. VI, 58, 59, 60, 61, 62, 63

Pius pp. VII, 64, 65, 66, 67, 68

19 Nomen loci per autores non inventum est.

La Personalidad Internacional de los Papas

Hispania Sacra 48 (1996) 
Plaza, Petrus, comendator domus et Hospitalis S. Antoni Abbatis Valentiae, 54

Pons, Petrus, rector eccl. parroch. loci de Manuel, Valentin. dioc., 43, 44

Portugalia, Agneti de, 16

Proxida, Elionor de, comitissa Aversae, 22

Proxida, Johanes Franciscus de, comes Almenarae et Aversae, 22

Pruno, Hieronimus de, pres. Valentin., 42

Ras, Bernardus, pres. Valentin., 9

Ribelles, Hieronymus de, can. Valentin., 29

Riganti, Nicolaus, in utraque signatura refer., 59

Robert, Ysabel, 22

Rodrigo, Bernardus, pres. Valentin., 15

Rodriguez, Vincentius, cler. Valentin., 46

Roig, Bernardus Johannes, cler. Valentin., 24, 25

Rupebertinus, Bernardus Hugonis de, castellanus Empostae, 19

Sala, Ludovicus, pres. Valentin., 9

Salt, Jacobus, 47

S. Antonius abbas, mon. extra muros Valentiae

Hospitalis, 54

Praeceptoria, 47

S. Bemardus, mon. extra muros Valentiae, ord. cisterc., abbas, $1,9,15$

S. Catherina Valentiae, eccl., 34

S. Crucis Valentiae, eccl. parroch., 14, 33

S. Franciscus, mon. Valentin., 28

S. Johannes de Mercato Valentiae, eccl., 45

S. Martinus Valentiae, eccl., 42

S. Michaelis de Cardona, eccl. parroch., Urgellen. dioc., 5

S. Quiteria, mon. ord. S. Benedicti, Aduren dioc., 18

S. Trinitas, mon. extra muros Hispalen., ministrus, 28

Segobricensis

Ep., 52, 53

Mensa ep., 68

Offic., $1,42,44,52,53$

Segura, Catherina de, ord. S. Bernardi cisterc., 30

Sixtus pp. IV, 18, 19, 20, 21

Sixtus pp. V, 35, 36, 37, 38

Stephani, Johannes, pres. Valentin., 14

Tarraconensis, 5, 33

Tomado, Marcus Antonius, in utraque signatura refer., 49

Ulldecona, Dertusen. dioc., baiulia seu praeceptoria, 19

Urbanus pp. VIII, 47 
Valença, Stephanus, 3, 4

Valenti, Johannes Gabrielis, cler. Valentin., 45

Valentia, Valentinus, $1,2,3,4,7,8,9,13,15,17,18,19,22,23,27,28,29,31,32$, $34,35,38,45,46,47,48,53,54,55,56,57,58,59,60,61,62,63,64,65,66,67$, 68, 69

Archidiac., 19

Archiep., 47, 49, 52, 59

Ep., 9, 10

Mensa archiep., 48, 53, 55, 56, 58, 59, 61, 62, 64, 65

Offic., $17,18,25,32,33,34,35,36,4 \mathrm{I}, 43,44,45,46,47,49,50,52,59$

Regnum, 18, 38

Valleriola, Arnardus, 34

Vallis digna, mon, cisterc. dioc. Valentin., 1

Villa Joyosa, Valentin. dioc., praeceptoria, 16

Vinader, Franciscus, cler. Valentin., 24, 25

Xenodochium Generalis Valentiae, vid. Hospitalis Generalis Valentiae

Yvanes, Jacobus, 32

Yvanyes, Petrus, cler. Valentin., 35

\section{INDEX ABBREVIATURARUM}

a.

A.D.P.

Aduren.

Albanen.

Andegaven.

ang.

archidiac.

archiep.

B.

Barchinonen.

Basilien.

ben.

can.

card.

cisterc.

cler.

cons.

La Personalidad Internacional de los Papas Hispania Sacra 48 (1996)

\section{annus}

Archivo de la Diputación de Valencia

Adurensis (dioc.)

Albanensis (dioc.)

Andegavensis (dioc.)

angulus

archidiaconus

archiepiscopus/archiepiscopalis

Beatus/Beati

Barchinonensis (dioc.)

Basiliensis (cons.)

beneficiatus

canapis-canonicus

cardinalis

cisterciensis

clericus

consilium 
cop. (not.)

Corduben.

d.

Dat.

dec.

dep.

Dertusen.

dioc.

doc.

eccl. (parroch.)

ep.

f./ff.

her.

Hispalen.

id.

Imolen.

in col.

inc. dom.

inf.

ins. in publ. instr

kal.

leg.

lin.

marg.

Malacitan.

mgcus.

mon.

Neucasstren.

non.

not.

offic.

ord.

orig.

Oriolen.

perg.

pont.

pp.

praep.

pres.

publ. copia (notarii)

Cordubensis (dioc.)

dextra

datum

decanus

deperditus

Dertusensis (dioc.)

diocesis

documentum

ecclesia (parrochialis)

episcopus/episcopalis

folius/folii

heremita

Hispalensis (dioc.)

idus

Imolensis (dioc.)

in columna

incarnatio dominica

inferior

insertum in publicum instrumentum

kalendae

legajo

linea

margo

Malacitanus

magnificus

monasterium

Neucastrensis (dioc.)

nonae

notarius

officialis

ordo

originale

Oriolensis (dioc.)

pergamino/s

pontificatus

Papa

praepositus

presbiter

publicum

La Personalidad Internacional de los Papas Hispania Sacra 48 (1996) 
r.

refer.

reg.

s.

S. SS

s.d

Segobricen.

ser.

sign.

sup.

Tarraconen.

vic.

Viennen. rectus

referendarius

regula/regularis

sinistra-saeculum

Sanctus/Sancti

sine data

Segobricensis (dioc.)

sericum

signatura

superior

Tạrraconensis (dioc.)

vicarius

Viennensis (dioc.)

\section{APPENDICES}

\section{APPENDIX}

\begin{tabular}{|r|l|l|c|}
\hline N. $^{\circ}$ & \multicolumn{1}{|c|}{ PAPAE } & \multicolumn{1}{|c|}{ DIMENSIONES $^{20}$} & SIGILUM \\
\hline 3 & Benedictus XIII & $500 \times 300+60$ & \\
\hline 5 & Benedictus XIII & $540 \times 299+42$ & \\
\hline 6 & Benedictus XIII & $514 \times 273+61$ & \\
\hline 7 & Benedictus XIII & $488 \times 273+62$ & \\
\hline 8 & Benedictus XIII & $665 \times 405+78$ & \\
\hline 9 & Benedictus XIII & $540 \times 346+56$ & \\
\hline 13 & Eugenius IV & $518 \times 310+66$ & \\
\hline 14 & Nicolaus V & $481 \times 261+83$ & \\
\hline 15 & Nicolaus V & $559 \times 286+43$ & \\
\hline 16 & Nicolaus V & $848 \times 565+72$ & \\
\hline 17 & Calistus III & $485 \times 300+55$ & \\
\hline 24 & Alexander VI & $512 \times 340+42$ & \\
\hline 25 & Alexander VI & $456 \times 270+50$ & \\
\hline 27 & Leo X & $953 \times 835+53$ & \\
\hline 32 & Gregorius XIII & $498 \times 300+67$ & \\
\hline
\end{tabular}

20 Documentorum latitudo $x$ altitudo + plica,

La Personalidad Internacional de los Papas

Hispania Sacra 48 (1996) 


\begin{tabular}{|c|c|c|c|}
\hline $\mathbf{N}^{*}$ & PAPAE & DIMENSIONES & SIGILUM \\
\hline 33 & Gregorius XIIII & $521 \times 325+64$ & $\mathrm{X}$ \\
\hline 34 & Gregorius XIII & $646 \times 439+56$ & \\
\hline 35 & Sixtus V & $416 \times 240+72$ & \\
\hline 37 & Sixtus V & $460 \times 245$ (breve) & \\
\hline 38 & Sixtus V & $737 \times 488+104$ & \\
\hline 42 & Paulus V & $368 \times 225+46$ & $\mathrm{X}$ \\
\hline 43 & Paulus V & $550 \times 328+76$ & \\
\hline 44 & Paulus V & $382 \times 288+44$ & \\
\hline 45 & Paulus V & $512 \times 311+76$ & \\
\hline 46 & Paulus V & $521 \times 311+64$ & \\
\hline 47 & Urbanus VIII & $582 \times 380+66$ & $\mathrm{X}$ \\
\hline 48 & Alexander VII & $563 \times 408+48$ & $\mathrm{x}$ \\
\hline 50 & Innocentius XII & $389 \times 230$ (breve) & $\mathrm{X}$ \\
\hline 51 & Innocentius XIII & $696 \times 445+98$ & $\mathrm{X}$ \\
\hline 52 & Innocentius XIII & $450 \times 296+6 \mathrm{I}$ & $\mathrm{X}$ \\
\hline 53 & Benedictus XIII & $417 \times 270+60$ & \\
\hline 54 & Benedictus XIII & $638 \times 410+63$ & \\
\hline 55 & Clemens XII & $429 \times 297$ (breve) & \\
\hline 56 & Benedictus XIV & $500 \times 375$ (breve) & $\mathrm{X}$ \\
\hline 57 & Clemens XIII & $400 \times 300$ (breve) & $\mathrm{X}$ \\
\hline 58 & Pius VI & $764 \times 500+80$ & \\
\hline 59 & Pius VI & $563 \times 377+65$ & \\
\hline 60 & Pius VI & $490 \times 312$ (breve) & \\
\hline 62 & Pius VI & $510 \times 330$ (breve) & $\ddot{x}$ \\
\hline 64 & Pius VII & $482 \times 334$ (breve) & $\mathrm{X}$ \\
\hline 65 & Pius VII & $490 \times 330$ (breve) & $\mathrm{X}$ \\
\hline 66 & Pius VII & $480 \times 342$ (breve) & $\mathrm{X}$ \\
\hline 67 & Pius VII & $775 \times 438+87$ & $\mathrm{X}$ \\
\hline 68 & Pius VII & $784 \times 627+51$ & $\mathrm{X}$ \\
\hline
\end{tabular}


TABULA DOCUMENTORUM

\begin{tabular}{|c|c|c|c|c|c|c|}
\hline Doc. & Orig. & $\begin{array}{c}\text { Cop. } \\
\text { simplex }\end{array}$ & Cop. not. & $\begin{array}{c}\text { Ins. in } \\
\text { publ. instr. }\end{array}$ & $\begin{array}{c}\text { cop. in } \\
\text { bullario }\end{array}$ & $\begin{array}{c}\text { Cop. in } \\
\text { chartulario }\end{array}$ \\
\hline 1 & & & & $X$ & & $X$ \\
\hline 2 & & & & & & $X$ \\
\hline 3 & $\mathrm{X}$ & & & & & \\
\hline 4 & & & & & & $\mathrm{X}$ \\
\hline 5 & $\mathrm{X}$ & & & & & \\
\hline 6 & $\mathrm{X}$ & & & & & \\
\hline 7 & $\mathrm{X}$ & & & & & $\mathrm{X}$ \\
\hline 8 & $\mathrm{X}$ & & & & & $\mathrm{X}$ \\
\hline 9 & $\mathrm{X}$ & & & & & \\
\hline 10 & & & & $\mathrm{X}$ & & \\
\hline 11 & & & $\mathrm{X}$ & & & \\
\hline 12 & & & & $\mathrm{X}$ & & \\
\hline 13 & $\mathrm{X}$ & & & & & \\
\hline 14 & $\mathrm{X}$ & & & & & \\
\hline 15 & $\mathrm{X}$ & & & $\mathrm{X}$ & & \\
\hline 16 & $\mathrm{X}$ & & & & & \\
\hline 17 & $\mathrm{X}$ & & & & & \\
\hline 18 & & & $\mathrm{X}$ & & & \\
\hline 9 & & & & $\mathrm{X}$ & & \\
\hline 20 & & & & $\mathrm{X}$ & & \\
\hline 21 & & & & $\mathrm{X}$ & & \\
\hline 22 & & & $\mathrm{X}$ & & & \\
\hline 23 & & & $\mathrm{X}$ & & & \\
\hline 24 & $\mathrm{X}$ & & & $\mathrm{X}$ & & \\
\hline 25 & $\mathrm{X}$ & & & $\mathrm{X}$ & & \\
\hline 26 & & & & $\mathrm{X}$ & & \\
\hline 27 & $\mathrm{X}$ & $\mathrm{X}$ & $\mathrm{X}$ & & & \\
\hline 28 & & & & $\mathrm{X}$ & & \\
\hline 29 & & & & $\mathrm{X}$ & & \\
\hline 30 & & & & $\mathrm{X}$ & & \\
\hline 31 & & & $\mathrm{X}$ & & & \\
\hline
\end{tabular}

La Personalidad Internacional de los Papas Hispania Sacra 48 (1996) 


\begin{tabular}{|c|c|c|c|c|c|c|}
\hline Doc. & Orig. & $\begin{array}{c}\text { Cop. } \\
\text { simplex }\end{array}$ & Cop. not. & $\begin{array}{c}\text { Ins. in } \\
\text { publ. instr. }\end{array}$ & $\begin{array}{c}\text { cop. in } \\
\text { bullario }\end{array}$ & $\begin{array}{c}\text { Cop. in } \\
\text { chartulario }\end{array}$ \\
\hline 32 & $\mathrm{X}$ & & & & & \\
\hline 33 & $\mathrm{X}$ & & & & & \\
\hline 34 & $\mathrm{X}$ & & & & & \\
\hline 35 & $\mathrm{X}$ & & & & & \\
\hline 36 & & & $\mathrm{X}$ & & & \\
\hline 37 & $\mathrm{X}$ & & & & & \\
\hline 38 & $\mathrm{X}$ & & & & & \\
\hline 39 & & & & $\mathrm{X}$ & & \\
\hline 40 & & & & $\mathrm{X}$ & & \\
\hline 41 & & & $\mathrm{X}$ & & & \\
\hline 42 & $\mathrm{X}$ & & & & & \\
\hline 43 & $\mathrm{X}$ & & & $\mathrm{X}$ & & \\
\hline 44 & $\mathrm{X}$ & & & & & \\
\hline 45 & $\mathrm{X}$ & & & & & \\
\hline 46 & $\mathrm{X}$ & & & & & \\
\hline 47 & $\mathrm{X}$ & & & & & \\
\hline 48 & $\mathrm{X}$ & & & & & \\
\hline 49 & & & $\mathrm{X}$ & & & \\
\hline 50 & $\mathrm{X}$ & & & & & \\
\hline 51 & $\mathrm{X}$ & & & & & \\
\hline 52 & $\mathrm{X}$ & & & & & \\
\hline 53 & $\mathrm{X}$ & & & & & \\
\hline 54 & $\mathrm{X}$ & & & & & \\
\hline 55 & $\mathrm{X}$ & & & & & \\
\hline 56 & $\mathrm{X}$ & & & & & \\
\hline 57 & $\mathrm{X}$ & $\mathrm{X}$ & & & & \\
\hline 58 & $\mathrm{X}$ & & & & $\mathrm{X}$ & \\
\hline 59 & $\mathrm{X}$ & & & & $\mathrm{X}$ & \\
\hline 60 & $\mathrm{X}$ & & & & $\mathrm{X}$ & \\
\hline 61 & & & & & $\mathrm{X}$ & \\
\hline 62 & $\mathrm{X}$ & & & & & \\
\hline 63 & & & & & & \\
\hline 64 & $\mathrm{X}$ & & & & & \\
\hline
\end{tabular}

La Personalidad Internacional de los Papas Hispania Sacra 48 (1996) 


\begin{tabular}{|c|c|c|c|c|c|c|}
\hline Doc. & Orig. & $\begin{array}{c}\text { Cop. } \\
\text { simplex }\end{array}$ & Cop. not. & $\begin{array}{c}\text { Ins. in } \\
\text { publ. instr. }\end{array}$ & $\begin{array}{c}\text { cop. in } \\
\text { bullario }\end{array}$ & $\begin{array}{c}\text { Cop. in } \\
\text { chartulario }\end{array}$ \\
\hline 65 & $\mathrm{X}$ & & & & $\mathrm{X}$ & \\
\hline 66 & $\mathrm{X}$ & & & & $\mathrm{X}$ & \\
\hline 67 & $\mathrm{X}$ & & & & & \\
\hline 68 & $\mathrm{X}$ & & & & $\mathrm{X}$ & \\
\hline 69 & & & & & $\mathrm{X}$ & \\
\hline
\end{tabular}

\section{BIBLIOGRAPHIA}

APARICIO Olmos, EMILIO MARIA: Santa María de los lnocentes y Desamparados en su iconografia original y sus precedentes históricos. Valencia, 1968.

CISCAR VILATA, CONCEPCION: El Hospital General de Valencia en el siglo XVIII (1700-1800): el edificio, el gobierno, el personal y la asistencia. Tesis doctoral. Valencia, 1992. Publicada en microficha, Valencia, 1994.

DOMINGO SIMÓ, FERNANDO y CALATAYUD BAYÁ, JOSÉ: El primer psiquiátrico del mundo. Valencia, 1959.

ESPLUGUES, IGNACIO: Relación y noticia de la fundación del Hospital Real y Ge. neral de la ciudad de Valencia. Valencia, 1793.

Gallent MarCo, MERCEDES: La asistencia sanitaria en Valencia (1400-15/2). Tesis doctoral inédita. Valencia, 1980.

GIMÉNEZ VALDIVIESO, EDUARDO: El Hospital de Valencia: informe emitido acerca de su origen, carticter y derecho exclusivo al disfrute de sus bienes. Valencia, 1907.

LÓPEZ TERRADA, MARÍA LuZ: El Hospital General de Valencia en el siglo XVI (1512-1600). Tesis doctoral. Valencia, 1986. Publicada en microficha, Valencia, 1987.

Peset, Mariano y PeSET, José Luis: "Felipe V y el Hospital Real y General de Valencia". En Medicina española, t. 61. Valencia 1969, pp. 405-414.

RODRIGO PERTEGÁs, JosÉ: Obra histórica de la Antigua Cofradía de Ntra. Sra. de los Inocentes Mártires y Desamparados, de la venerada imagen y de su capilla. Valencia, 1922.

SEMPERE CORBí, JoRge: Cómo nació, cómo era, cómo funcionaba el "Hospital dels folls de Sancta Maria dels Ignoscents". Valencia, 1959.

TROPE, HÉlÉnE: Locura y sociedad en la Valencia de los siglos XV al XVII. Valencia, 1995.

VILAR DEVIS, MARÍA MERCEDES: El Hospital General de Valencia en el siglo XVII $(1600-1700)$. Tesis doctoral inédita. Valencia, 1990.

\footnotetext{
La Personalidad Internacional de los Papas Hispania Sacra 48 (1996)
} 


\section{GRAPHICAE}

Distributio inter originalia et copias

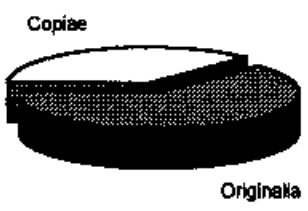

Distributio documentorum per archiva

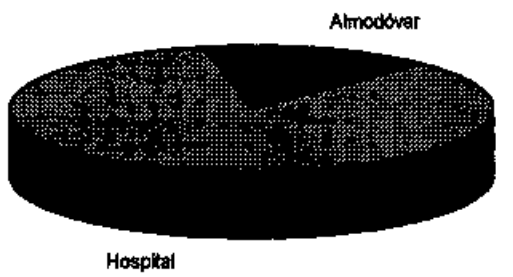

Distributio documentorum per pontifices

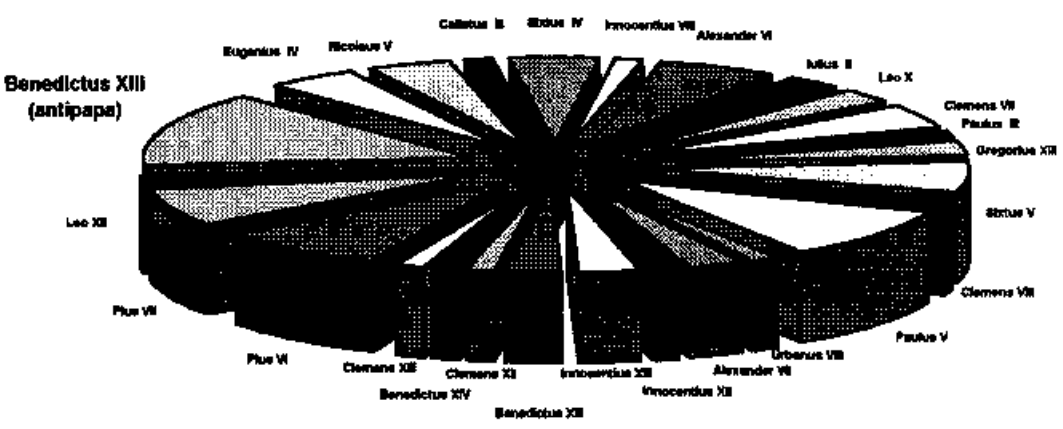

La Personalidad Internacional de los Papas Hispania Sacia 48 (1996) 